\title{
Quantization for a fourth order equation with critical exponential growth
}

\author{
Michael Struwe
}

Received: 6 March 2006 / Accepted: 6 September 2006 /

Published online: 22 December 2006

(C) Springer-Verlag 2006

Abstract For concentrating solutions $0<u_{k} \rightarrow 0$ weakly in $H^{2}(\Omega)$ to the equation $\Delta^{2} u_{k}=\lambda_{k} u_{k} e^{2 u_{k}^{2}}$ on a domain $\Omega \subset \mathbb{R}^{4}$ with Navier boundary conditions the concentration energy $\Lambda=\lim _{k \rightarrow \infty} \int_{\Omega}\left|\Delta u_{k}\right|^{2} d x$ is shown to be strictly quantized in multiples of the number $\Lambda_{1}=16 \pi^{2}$.

\section{Introduction}

Let $\Omega$ be a bounded domain of $\mathbb{R}^{4}$ and let $u_{k}>0$ be solutions to the equation

$$
\Delta^{2} u_{k}=\lambda_{k} u_{k} e^{2 u_{k}^{2}} \quad \text { in } \Omega
$$

with constants $\lambda_{k}>0$, where we prescribe Navier boundary conditions

$$
u_{k}=\Delta u_{k}=0 \quad \text { on } \partial \Omega .
$$

We assume that $\lambda_{k} \rightarrow 0$ and

$$
\int_{\Omega}\left|\Delta u_{k}\right|^{2} \mathrm{~d} x=\int_{\Omega} u_{k} \Delta^{2} u_{k} \mathrm{~d} x=\lambda_{k} \int_{\Omega} u_{k}^{2} e^{2 u_{k}^{2}} \mathrm{~d} x \rightarrow \Lambda>0
$$

as $k \rightarrow \infty$. In view of the boundary condition $u_{k}=0$ on $\partial \Omega$, then by standard elliptic estimates we also have the uniform estimate

$$
\int_{\Omega}\left|\nabla^{2} u_{k}\right|^{2} \mathrm{~d} x \leq C \int_{\Omega}\left|\Delta u_{k}\right|^{2} \mathrm{~d} x \leq C
$$

for all $k$. Since $\lambda_{k} \rightarrow 0$, from (3), (4) we conclude that $\Delta^{2} u_{k} \rightarrow 0$ in $L^{1}(\Omega)$ and $u_{k} \rightarrow 0$ weakly in $H^{2}(\Omega)$ as $k \rightarrow \infty$, but not strongly. In fact, as shown in [10], the sequence

M. Struwe $(\bowtie)$

Departement für Mathematik, ETH-Zentrum, 8092 Zürich, Switzerland

e-mail: struwe@math.ethz.ch 
$\left(u_{k}\right)$ blows up in a finite number of points where after rescaling spherical bubbles form in the following sense.

Theorem 1.1 Let $\Omega$ be a bounded domain of $\mathbb{R}^{4}$ and let $\left(u_{k}\right)_{k \in \mathbb{N}}$ be a sequence of positive solutions to problem (1), (2), satisfying (3) for some $\Lambda>0$ as above.

Then there exist a subsequence $\left(u_{k}\right)$ and finitely many points $x^{(i)} \in \Omega, 1 \leq i \leq$ $I \leq C \Lambda$, such that for each $i$ with suitable points $x_{k}=x_{k}^{(i)} \rightarrow x^{(i)}$ and scale factors $0<r_{k}=r_{k}^{(i)} \rightarrow 0$ satisfying

$$
\lambda_{k} r_{k}^{4} u_{k}^{2}\left(x_{k}\right) e^{2 u_{k}^{2}\left(x_{k}\right)}=96
$$

we have

$$
\eta_{k}(x)=\eta_{k}^{(i)}(x):=u_{k}\left(x_{k}\right)\left(u_{k}\left(x_{k}+r_{k} x\right)-u_{k}\left(x_{k}\right)\right) \rightarrow \eta_{0}=\log \left(\frac{1}{1+|x|^{2}}\right)
$$

locally $C^{3}$-uniformly on $\mathbb{R}^{4}$ as $k \rightarrow \infty$, where $\eta=\eta_{0}+\log 2=\log \left(\frac{2}{1+|x|^{2}}\right)$ solves the fourth order analogue of Liouville's equation

$$
\Delta^{2} \eta=\Delta^{2} \eta_{0}=96 e^{4 \eta_{0}}=6 e^{4 \eta} \quad \text { on } \mathbb{R}^{4} .
$$

In addition we have

$$
\frac{\left|x_{k}^{(i)}-x_{k}^{(j)}\right|}{r_{k}^{(i)}} \rightarrow \infty \quad \text { for all } 1 \leq i \neq j \leq I,
$$

and there holds the pointwise estimate

$$
\lambda_{k} \inf _{i}\left|x-x_{k}^{(i)}\right|^{4} u_{k}^{2}(x) e^{2 u_{k}^{2}(x)} \leq C,
$$

uniformly for all $x \in \Omega$ and all $k$.

Geometrically, the solutions $\eta$ to the limit equation (7) correspond to conformal metrics $g=e^{2 \eta} g_{\mathbb{R}^{4}}$ on $\mathbb{R}^{4}$ of constant $Q$-curvature $Q=\frac{1}{2} e^{-4 \eta} \Delta^{2} \eta=3=Q_{S^{4}}$, which are obtained by pull-back of the spherical metric on $S^{4}$ under stereographic projection and with total $Q$-curvature

$$
2 \int_{\mathbb{R}^{4}} Q \mathrm{~d} \mu_{g}=\int_{\mathbb{R}^{4}} 6 e^{4 \eta} \mathrm{d} x=2 \int_{S^{4}} Q_{S^{4}} \mathrm{~d} \mu_{g_{S^{4}}}=16 \pi^{2}=: \Lambda_{1} .
$$

This geometric interpretation of $\eta$ is the reason why we prefer to state the preceding result in the present form rather than choosing scaling constants as in [10].

Continuing our previous work, here we show that the concentration energy $\Lambda$ is quantized in multiples of $\Lambda_{1}$.

Theorem 1.2 In the context of Theorem 1.1 we have $\Lambda=L \Lambda_{1}$ for some $L \in \mathbb{N}$.

Theorem 1.2 is the four-dimensional analogue of a recent result by Druet [4] for the corresponding 2-dimensional equation

$$
-\Delta u_{k}=\lambda_{k} u_{k} e^{2 u_{k}^{2}} \quad \text { in } \Omega \subset \mathbb{R}^{2},
$$


which refines our previous result with Adimurthi in [2], characterizing only the first blow-up energy level.

A similar quantization has been observed by Wei [11] for the fourth order analogue of Liouville's equation

$$
\Delta^{2} u_{k}=\lambda_{k} e^{4 u_{k}} \quad \text { in } \Omega \subset \mathbb{R}^{4},
$$

with Navier boundary conditions (2), assuming the uniform $L^{1}$-bound

$$
\int_{\Omega} \lambda_{k} e^{4 u_{k}} \mathrm{~d} x \leq \Lambda
$$

and with $\lambda_{k} \rightarrow 0$ as $k \rightarrow \infty$. Quite remarkably, Wei is able to show that for (12) each blow-up point is simple in the sense that $L=I$. In the geometric context of the problem of prescribed $Q$-curvature on $S^{4}$, an analogous result was obtained by Malchiodi and this author [8]. It is an interesting open question whether the same strong quantization property holds true for Eq. (1) as well.

Related results on compactness issues for fourth order equations can be found in $[1,5,9]$, or [7].

In the following two sections we first present the proof of Theorem 1.2 in the rotationally symmetric case. In Sect. 4 then we show how these results can be extended to the general case by means of the gradient estimate Proposition 4.1, whose proof is given in Sect. 5. Clearly, we may pass to further subsequences $\left(u_{k}\right)$, when needed. Throughout we let $\Delta=\sum_{i=1}^{4}\left(\partial / \partial x_{i}\right)^{2}$ be the Laplacian (with the analysts'sign). The letter $C$ denotes a generic constant that may change from line to line.

I thank Olivier Druet for helpful comments on an early version of this paper.

\section{The radial case}

The proof of Theorem 1.2 is most transparent in the radial case where $\Omega=B_{R}=B_{R}(0)$ and when $u_{k}(x)=u_{k}(|x|)$. Indeed, in this case in the notations of Theorem 1.1 we have $I=1, x_{k}^{(1)}=x^{(1)}=0$, and

$$
\eta_{k}(x)=\eta_{k}^{(1)}(x)=u_{k}(0)\left(u_{k}\left(r_{k} x\right)-u_{k}(0)\right) \leq 0,
$$

where $r_{k}=r_{k}^{(1)}$ is given by (5). Moreover, the functions $u_{k}$ and $-\Delta u_{k}$ are positive and radially decreasing, and $u_{k} \rightarrow 0$ locally uniformly away from $x=0$ as $k \rightarrow \infty$.

For brevity we let

$$
u_{k} \Delta^{2} u_{k}=\lambda_{k} u_{k}^{2} e^{2 u_{k}^{2}}=: e_{k} \quad \text { in } \Omega
$$

and we denote as

$$
w_{k}(x)=u_{k}(0)\left(u_{k}(x)-u_{k}(0)\right)
$$

the unscaled function $\eta_{k}$, satisfying the equation

$$
\begin{aligned}
\Delta^{2} w_{k} & =\lambda_{k} u_{k}(0) u_{k} e^{2 u_{k}^{2}} \\
& =\lambda_{k}\left(u_{k}^{2}(0)+w_{k}\right) e^{2 u_{k}^{2}(0)} e^{4\left(1+\frac{w_{k}}{2 u_{k}^{2}(0)}\right) w_{k}}=: f_{k} \geq e_{k} \quad \text { in } \Omega=B_{R} .
\end{aligned}
$$


The function $\eta_{k}$ likewise satisfies the equation

$$
\Delta^{2} \eta_{k}=V_{k} e^{4 a_{k} \eta_{k}} \quad \text { in } \Omega_{k}=B_{R / r_{k}}(0)
$$

with

$$
0 \leq V_{k}=\lambda_{k} r_{k}^{4}\left(u_{k}^{2}(0)+\eta_{k}\right) e^{2 u_{k}^{2}(0)} \leq 96,1 / 2 \leq a_{k}=1+\frac{\eta_{k}}{2 u_{k}^{2}(0)} \leq 1 .
$$

In view of Theorem 1.1 we have $V_{k} \rightarrow 96, a_{k} \rightarrow 1$ locally uniformly on $\mathbb{R}^{4}$ as $k \rightarrow \infty$.

For $0<r<R$ let

$$
\Lambda_{k}(r)=\int_{B_{r}} e_{k} \mathrm{~d} x, \quad \sigma_{k}(r)=\int_{B_{r}} f_{k} \mathrm{~d} x
$$

Observe that $\Lambda_{k}(r) \leq \sigma_{k}(r)$, and both functions are non-decreasing in $r$; moreover, (10) implies

$$
\lim _{L \rightarrow \infty} \lim _{k \rightarrow \infty} \Lambda_{k}\left(L r_{k}\right)=\lim _{L \rightarrow \infty} \lim _{k \rightarrow \infty} \sigma_{k}\left(L r_{k}\right)=\lim _{L \rightarrow \infty} \lim _{k \rightarrow \infty} 96 \int_{B_{L}} e^{4 \eta_{k}} \mathrm{~d} x=\Lambda_{1}
$$

We can now show our first decay estimate.

Lemma 2.1 For any $\varepsilon>0$, letting $T_{k}>0$ be such that $u_{k}\left(T_{k}\right)=\varepsilon u_{k}(0) / 2$, for any constant $b<2$ and sufficient large $k$ there holds

$$
w_{k}(r) \leq b \log \left(\frac{r_{k}}{r}\right)+C \quad \text { on } B_{T_{k}}
$$

and we have

$$
\lim _{k \rightarrow \infty} \Lambda_{k}\left(T_{k}\right)=\lim _{k \rightarrow \infty} \sigma_{k}\left(T_{k}\right)=\Lambda_{1}
$$

Proof Note that $T_{k} \rightarrow 0$ as $k \rightarrow \infty$ in view of the locally uniform convergence $u_{k} \rightarrow 0$ away from 0 .

For $0 \leq t \leq R$ decompose $w_{k}=g_{k}+h_{k}$ on $B_{t}$, where $g_{k}$ satisfies $g_{k}=\partial_{\nu} g_{k}=0$ on $\partial B_{t}$ and where $\Delta^{2} h_{k}=0$. Then we have $\Delta h_{k} \equiv 8 d_{k}$ for some $d_{k} \in \mathbb{R}$ and it follows that $h_{k}(x)=w_{k}(t)+d_{k}\left(|x|^{2}-t^{2}\right)$. The equations $\partial_{\nu} w_{k}=\partial_{\nu} g_{k}+\partial_{\nu} h_{k}=2 t d_{k}$ and $\Delta w_{k}=\Delta g_{k}+\Delta h_{k}=\Delta g_{k}+8 d_{k}$ on $\partial B_{t}$ then imply the identity

$$
\Delta w_{k}-4 t^{-1} \partial_{\nu} w_{k}=\Delta g_{k} \quad \text { on } \partial B_{t} .
$$

If we now choose $t=t_{k} \geq L r_{k}$ from (17) we obtain

$$
\begin{aligned}
\int_{\partial B_{t}} t \Delta g_{k} \mathrm{~d} o & =\int_{\partial B_{t}} \partial_{\nu}\left(x \cdot \nabla g_{k}\right) \mathrm{d} o=\int_{B_{t}}\left(2 \Delta g_{k}+x \cdot \nabla \Delta g_{k}\right) \mathrm{d} x \\
& =\int_{B_{t}} x \cdot \nabla \Delta g_{k} \mathrm{~d} x=\int_{0}^{t} r \int_{\partial B_{r}} \partial_{\nu} \Delta g_{k} \mathrm{~d} o \mathrm{~d} r=\int_{0}^{t} r \sigma_{k}(r) \mathrm{d} r \\
& \geq \frac{t^{2}}{2}\left(\Lambda_{1}-o(1)\right)
\end{aligned}
$$


with error $o(1) \rightarrow 0$ if first $k \rightarrow \infty$ and then $L \rightarrow \infty$. For any $b<2$ and sufficiently large $L \geq L(b)$ for $k \geq k_{0}(L)$ then we conclude the estimate

$$
\Delta g_{k} \geq \frac{1}{2 \omega_{3} t^{2}}\left(\Lambda_{1}-o(1)\right) \geq \frac{2 b}{t^{2}} \quad \text { on } \partial B_{t}
$$

for such $t=t_{k}$, where $\omega_{3}=2 \pi^{2}$ is the volume of $S^{3}$. Writing $w_{k}(x)=w_{k}(r)$ for convenience and denoting $w_{k}^{\prime}=\frac{\partial w_{k}}{\partial r}$ and so on, we thus obtain that

$$
r\left(\frac{w_{k}^{\prime}}{r}+\frac{b}{r^{2}}\right)^{\prime}=w_{k}^{\prime \prime}-\frac{w_{k}^{\prime}}{r}-\frac{2 b}{r^{2}}=\Delta w_{k}-4 r^{-1} \partial_{\nu} w_{k}-\frac{2 b}{r^{2}} \geq 0
$$

on $\partial B_{t}$, provided $t \geq L r_{k}$ with $L \geq L(b)$ and $k$ sufficiently large; that is, the expression $\frac{w_{k}^{\prime}}{r}+\frac{b}{r^{2}}$ is non-decreasing for $r \geq L r_{k}$. Since $w_{k}^{\prime} \leq 0$ we conclude that

$$
w_{k}^{\prime}(r) \leq-\frac{b}{r}+\frac{r w_{k}^{\prime}(t)}{t}+\frac{r b}{t^{2}} \leq-\frac{b}{r}+\frac{r b}{t^{2}}
$$

for all $t=t_{k} \geq r \geq L r_{k}$. For any $r \in\left[L r_{k}, T_{k}\right]$, upon choosing $t=t_{k}$ where $T_{k} / t_{k} \rightarrow 0$ as $k \rightarrow \infty$ and integrating from $L r_{k}$ to $r$, with error $o(1) \rightarrow 0$ as $k \rightarrow \infty$ from (6) we find

$$
\begin{aligned}
w_{k}(r) & \leq w_{k}\left(L r_{k}\right)-b \log \left(\frac{r}{L r_{k}}\right)+o(1) \\
& =\eta_{k}(L)+b \log L+b \log \left(\frac{r_{k}}{r}\right)+o(1) \leq b \log \left(\frac{r_{k}}{r}\right)+o(1),
\end{aligned}
$$

as claimed in (18). For $r \leq L r_{k}$ the asserted bound already follows from Theorem 1.1.

Inserting (18) in the definition (14) of $f_{k}$ and recalling (5), for $L r_{k} \leq r \leq T_{k}$ with sufficiently large $L>0$ and $k$ then we obtain

$$
\begin{aligned}
f_{k} & \leq \lambda_{k} u_{k}^{2}(0) e^{2 u_{k}^{2}(0)} e^{4\left(1+\frac{w_{k}}{2 u_{k}^{2}(0)}\right) w_{k}} \\
& \leq \lambda_{k} r_{k}^{4} u_{k}^{2}(0) e^{2 u_{k}^{2}(0)} r_{k}^{-4} e^{(2+\varepsilon) w_{k}} \leq C r_{k}^{-4}\left(\frac{r_{k}}{r}\right)^{(2+\varepsilon) b} .
\end{aligned}
$$

Choosing $b<2$ such that $(2+\varepsilon) b=4+\varepsilon$, upon integrating over $B_{T_{k}}$ we obtain

$$
\begin{aligned}
\sigma_{k}\left(T_{k}\right) & =\int_{B_{T_{k}}} f_{k} \mathrm{~d} x \leq \Lambda_{1}+\int_{B_{T_{k}} \backslash B_{L r_{k}}} f_{k} \mathrm{~d} x \\
& \leq \Lambda_{1}+C r_{k}^{-4} \int_{B_{T_{k}} \backslash B_{L r_{k}}}\left(\frac{r_{k}}{r}\right)^{4+\varepsilon} \mathrm{d} x \leq \Lambda_{1}+C \varepsilon^{-1}\left(\frac{r_{k}}{L r_{k}}\right)^{\varepsilon} \leq \Lambda_{1}+\varepsilon,
\end{aligned}
$$

if first $L>L_{0}(\varepsilon)$ and then $k \geq k_{0}(L)$ is chosen sufficiently large. Since $\varepsilon>0$ is arbitrary, the proof is complete.

For a suitable sequence $\varepsilon_{k} \downarrow 0$ as $k \rightarrow \infty$ and corresponding numbers $s_{k}=T_{k}\left(\varepsilon_{k}\right)$ then we have

$$
\lim _{k \rightarrow \infty} \Lambda_{k}\left(s_{k}\right)=\lim _{k \rightarrow \infty} \sigma_{k}\left(s_{k}\right)=\Lambda_{1}, \quad \lim _{k \rightarrow \infty} \frac{u_{k}\left(s_{k}\right)}{u_{k}\left(r_{k}\right)}=\lim _{k \rightarrow \infty} \frac{r_{k}}{s_{k}}=0,
$$


where $r_{k} / s_{k} \rightarrow 0$ as $k \rightarrow \infty$ by Theorem 1.1. In addition from (17) we obtain that

$$
\lim _{L \rightarrow \infty} \lim _{k \rightarrow \infty}\left(\Lambda_{k}\left(s_{k}\right)-\Lambda_{k}\left(L r_{k}\right)\right)=0
$$

Let $r_{k}=r_{k}^{(1)}, s_{k}=s_{k}^{(1)}$. We now proceed by iteration. Suppose that for some integer $l \geq 1$ we already have determined numbers $r_{k}^{(1)}<s_{k}^{(1)}<\cdots<r_{k}^{(l)}<s_{k}^{(l)}$ such that

$$
\lim _{k \rightarrow \infty} \Lambda_{k}\left(s_{k}^{(l)}\right)=l \Lambda_{1}
$$

and

$$
\lim _{L \rightarrow \infty} \lim _{k \rightarrow \infty}\left(\Lambda_{k}\left(s_{k}^{(l)}\right)-\Lambda_{k}\left(L r_{k}^{(l)}\right)\right)=\lim _{k \rightarrow \infty} \frac{u_{k}\left(s_{k}^{(l)}\right)}{u_{k}\left(r_{k}^{(l)}\right)}=\lim _{k \rightarrow \infty} \frac{r_{k}^{(l)}}{s_{k}^{(l)}}=0 .
$$

For $0<s<t<R$ let

$$
N_{k}(s, t)=\int_{B_{t} \backslash B_{s}} e_{k} \mathrm{~d} x=\int_{B_{t} \backslash B_{s}} \lambda_{k} u_{k}^{2} e^{2 u_{k}^{2}} \mathrm{~d} x=\omega_{3} \int_{s}^{t} \lambda_{k} r^{3} u_{k}^{2} e^{2 u_{k}^{2}} \mathrm{~d} r
$$

and define

$$
P_{k}(t)=t \frac{\partial}{\partial t} N_{k}(s, t)=t \int_{\partial B_{t}} e_{k} \mathrm{~d} o=\omega_{3} \lambda_{k} t^{4} u_{k}^{2}(t) e^{2 u_{k}^{2}(t)} .
$$

Note that monotonicity of $u_{k}$ implies

$$
\begin{aligned}
P_{k}(t) & =\omega_{3} \lambda_{k} t^{4} u_{k}^{2}(t) e^{2 u_{k}^{2}(t)}=C_{0} \omega_{3} \lambda_{k} u_{k}^{2}(t) e^{2 u_{k}^{2}(t)} \int_{t / 2}^{t} r^{3} \mathrm{~d} r \\
& \leq C_{0} N_{k}(t / 2, t) \leq C_{1} P_{k}(t / 2)
\end{aligned}
$$

with uniform constants $C_{0}=4(1-1 / 16)^{-1}, C_{1}=16$, respectively.

A preliminary quantification now can be achieved, as follows.

\section{Lemma 2.2}

(i) Suppose that for some $t_{k}>s_{k}^{(l)}$ there holds

$$
\sup _{\substack{(l) \\ s_{k}<t<t_{k}}} P_{k}(t) \rightarrow 0 \quad \text { as } k \rightarrow \infty \text {. }
$$

Then we have

$$
\lim _{k \rightarrow \infty} N_{k}\left(s_{k}^{(l)}, t_{k}\right)=0
$$

(ii) Conversely, if for some $t_{k}>s_{k}^{(l)}$ and a subsequence $\left(u_{k}\right)$ there holds

$$
\lim _{k \rightarrow \infty} N_{k}\left(s_{k}^{(l)}, t_{k}\right)=c_{0}>0,
$$

then either $c_{0} \geq 2 \omega_{3}=4 \pi^{2}$, or we have

$$
\liminf _{k \rightarrow \infty} P_{k}\left(t_{k}\right) \geq 2 c_{0}
$$

Springer 
and

$$
\lim _{L \rightarrow \infty} \liminf _{k \rightarrow \infty} N_{k}\left(s_{k}^{(l)}, L t_{k}\right) \geq 2 \omega_{3}, \quad \lim _{L \rightarrow \infty} \limsup _{k \rightarrow \infty} N_{k}\left(s_{k}^{(l)}, t_{k} / L\right)=0
$$

Proof (i) For $s=s_{k}^{(l)}<t$ we integrate by parts to obtain

$$
\begin{aligned}
4 N_{k}(s, t) & =4 \int_{B_{t} \backslash B_{s}} \lambda_{k} u_{k}^{2} e^{2 u_{k}^{2}} \mathrm{~d} x=4 \omega_{3} \int_{s}^{t} \lambda_{k} r^{3} u_{k}^{2} e^{2 u_{k}^{2} \mathrm{~d} r} \\
& =\left.\omega_{3} \lambda_{k}\left(r^{4} u_{k}^{2} e^{2 u_{k}^{2}}\right)\right|_{s} ^{t}-4 \omega_{3} \int_{s}^{t} \lambda_{k} r^{4}\left(u_{k} / 2+u_{k}^{3}\right) u_{k}^{\prime} e^{2 u_{k}^{2} \mathrm{~d} r} \\
& \leq P_{k}(t)-4 \omega_{3} \int_{s}^{t} \lambda_{k} r^{4}\left(1 / 2+u_{k}^{2}\right) \frac{u_{k}}{u_{k}(0)} w_{k}^{\prime} e^{2 u_{k}^{2}} \mathrm{~d} r .
\end{aligned}
$$

In order to further estimate the right hand side we need to derive a lower bound for $w_{k}^{\prime}$. For $0<r<t$ decompose $w_{k}=g_{k}+h_{k}$ on $B_{r}$ as in the proof of Lemma 2.1, where $g_{k}$ satisfies $g_{k}=\partial_{\nu} g_{k}=0$ on $\partial B_{r}$ and where $\Delta^{2} h_{k}=0$. Then

$$
\Delta w_{k}-4 r^{-1} \partial_{\nu} w_{k}=\Delta g_{k} \quad \text { on } \partial B_{r}
$$

as before while (20) yields the equation

$$
\Delta g_{k}(r)=\frac{\int_{\partial B_{r}} \Delta g_{k} \mathrm{~d} o}{\omega_{3} r^{3}}=\frac{\int_{0}^{r} r^{\prime} \sigma_{k}\left(r^{\prime}\right) \mathrm{d} r^{\prime}}{\omega_{3} r^{4}} \leq \frac{\sigma_{k}(r)}{2 \omega_{3} r^{2}} .
$$

We conclude that

$$
\begin{aligned}
& r\left(\frac{w_{k}^{\prime}}{r}+\frac{\int_{0}^{r} r^{\prime} \sigma_{k}\left(r^{\prime}\right) d r^{\prime}}{2 \omega_{3} r^{4}}\right)^{\prime} \\
& \quad=w_{k}^{\prime \prime}-\frac{w_{k}^{\prime}}{r}+\frac{\sigma_{k}(r)}{2 \omega_{3} r^{2}}-\frac{2 \int_{0}^{r} r^{\prime} \sigma_{k}\left(r^{\prime}\right) d r^{\prime}}{\omega_{3} r^{4}} \geq \Delta w_{k}-4 r^{-1} \partial_{\nu} w_{k}-\Delta g_{k}=0
\end{aligned}
$$

on $\partial B_{r}$. Thus, for $0 \leq r_{0} \leq r$ we have

$$
\frac{w_{k}^{\prime}(r)}{r}+\frac{\int_{0}^{r} r^{\prime} \sigma_{k}\left(r^{\prime}\right) \mathrm{d} r^{\prime}}{2 \omega_{3} r^{4}} \geq \frac{w_{k}^{\prime}\left(r_{0}\right)}{r_{0}}+\frac{\int_{0}^{r_{0}} r^{\prime} \sigma_{k}\left(r^{\prime}\right) \mathrm{d} r^{\prime}}{2 \omega_{3} r_{0}^{4}} .
$$

Upon choosing $r_{0}=L r_{k}$ for some fixed $L$, from Theorem 1.1 we can explicitly compute the limit as $k \rightarrow \infty$ of the term on the right to see that

$$
\frac{w_{k}^{\prime}(r)}{r}+\frac{\int_{0}^{r} r^{\prime} \sigma_{k}\left(r^{\prime}\right) \mathrm{d} r^{\prime}}{2 \omega_{3} r^{4}} \geq 0
$$

for $r \geq L r_{k}$ when $L$ and $k \geq k_{0}(L)$ are sufficiently large; that is, there holds

$$
r w_{k}^{\prime}(r) \geq-\frac{\int_{0}^{r} r^{\prime} \sigma_{k}\left(r^{\prime}\right) \mathrm{d} r^{\prime}}{2 \omega_{3} r^{2}} \geq-\frac{\sigma_{k}(r)}{4 \omega_{3}} \quad \text { for all } r \geq L r_{k} .
$$


Inserting the bound (28) into (27) and observing that by (25) for $s=s_{k}^{(l)}<r<t$ with error $o(1) \rightarrow 0$ as $k \rightarrow \infty$ we have

$$
\begin{aligned}
\frac{u_{k}(r)}{u_{k}(0)} \sigma_{k}(r) & =\omega_{3} \int_{0}^{r} \lambda_{k} u_{k}(r) u_{k}\left(r^{\prime}\right) e^{2 u_{k}^{2}\left(r^{\prime}\right)} r^{\prime 3} \mathrm{~d} r^{\prime} \\
& \leq \omega_{3} \int_{s}^{r} \lambda_{k} u_{k}^{2}\left(r^{\prime}\right) e^{2 u_{k}^{2}\left(r^{\prime}\right)} r^{\prime 3} \mathrm{~d} r^{\prime}+o(1)=N_{k}(s, r)+o(1),
\end{aligned}
$$

we arrive at the estimate

$$
\begin{aligned}
4 N_{k}(s, t) & \leq P_{k}(t)+\int_{s}^{t} \lambda_{k} r^{3}\left(1 / 2+u_{k}^{2}\right) e^{2 u_{k}^{2}} N_{k}(s, r) \mathrm{d} r+o(1) \\
& \leq P_{k}(t)+\omega_{3}^{-1} N_{k}(s, t)^{2}+o(1)
\end{aligned}
$$

If we now assume that

$$
\sup _{s<t<t_{k}} P_{k}(t) \rightarrow 0 \quad \text { as } k \rightarrow \infty
$$

upon letting $t$ increase from $t=s=s_{k}^{(l)}$ to $t_{k}$ we find

$$
\lim _{k \rightarrow \infty} N_{k}\left(s_{k}^{(l)}, t_{k}\right)=0,
$$

as claimed.

(ii) On the other hand, if we suppose that for some $t_{k}>s_{k}^{(l)}$ we have

$$
0<\lim _{k \rightarrow \infty} N_{k}\left(s_{k}^{(l)}, t_{k}\right)=c_{0}<2 \omega_{3},
$$

from (29) with error $o(1) \rightarrow 0$ as $k \rightarrow \infty$ we conclude that

$$
2 c_{0}+o(1) \leq\left(4-c_{0} \omega_{3}^{-1}\right) N_{k}\left(s_{k}^{(l)}, t_{k}\right) \leq P_{k}\left(t_{k}\right)+o(1) .
$$

It also follows that

$$
\lim _{L \rightarrow \infty} \liminf _{k \rightarrow \infty} N_{k}\left(s_{k}^{(l)}, L t_{k}\right) \geq 2 \omega_{3}
$$

Otherwise, (26) and (31) yield the uniform bound

$$
C_{0} \liminf _{k \rightarrow \infty} N_{k}\left(L t_{k} / 2, L t_{k}\right) \geq \liminf _{k \rightarrow \infty} P_{k}\left(L t_{k}\right) \geq 2 c_{0}
$$

for all $L \in \mathbb{N}$. Choosing $L=2^{m}$, where $m \in \mathbb{N}$, and summing over $1 \leq m \leq M$, we obtain

$$
C_{0} \liminf _{k \rightarrow \infty} \Lambda_{k}\left(2^{M} t_{k}\right) \geq C_{0} \liminf _{k \rightarrow \infty} N_{k}\left(t_{k}, 2^{M} t_{k}\right) \geq 2 c_{0} M \rightarrow \infty \quad \text { as } M \rightarrow \infty,
$$

contrary to assumption (3). Upon replacing $t_{k}$ by $t_{k} / L$ in the previous argument and recalling our assumption (30), by the same reasoning we also arrive at the estimate

$$
\lim _{L \rightarrow \infty} \liminf _{k \rightarrow \infty} N_{k}\left(s_{k}^{(l)}, t_{k} / L\right)=0 .
$$

This completes the proof. 
Suppose that for some $t_{k}>s_{k}^{(l)}$ there holds

$$
\liminf _{k \rightarrow \infty} N_{k}\left(s_{k}^{(l)}, t_{k}\right)>0
$$

Then we can find a subsequence $\left(u_{k}\right)$ and numbers $\left.r_{k}^{(l+1)} \in\right] s_{k}^{(l)}, t_{k}[$ such that

$$
\lim _{k \rightarrow \infty} N_{k}\left(s_{k}^{(l)}, r_{k}^{(l+1)}\right)=c_{0}>0 .
$$

Replacing our original choice of $r_{k}^{(l+1)}$ by a smaller number, if necessary, we may assume that $c_{0}<2 \omega_{3}$. Lemma 2.2 then implies that

$$
\lim _{L \rightarrow \infty} \liminf _{k \rightarrow \infty} N_{k}\left(s_{k}^{(l)}, L r_{k}^{(l+1)}\right) \geq 2 \omega_{3}, \quad \lim _{L \rightarrow \infty} \limsup _{k \rightarrow \infty} N_{k}\left(s_{k}^{(l)}, r_{k}^{(l+1)} / L\right)=0,
$$

and that

$$
\liminf _{k \rightarrow \infty} P_{k}\left(r_{k}^{(l+1)}\right)>0
$$

Note that (25) and (26) also yield that $r_{k}^{(l+1)} / s_{k}^{(l)} \rightarrow \infty$ as $k \rightarrow \infty$. Indeed, from (25) and (26) for any $m \in \mathbb{N}$ with error $o(1) \rightarrow 0$ as $k \rightarrow \infty$ we obtain that

$$
\begin{aligned}
o(1) & \geq N_{k}\left(s_{k}^{(l)} / 2, s_{k}^{(l)}\right) \geq C_{0}^{-1} P_{k}\left(s_{k}^{(l)}\right) \\
& \geq C_{1}^{-1} N_{k}\left(s_{k}^{(l)}, 2 s_{k}^{(l)}\right) \geq \cdots \geq C_{1}^{-m} N_{k}\left(2^{m-1} s_{k}^{(l)}, 2^{m} s_{k}^{(l)}\right) .
\end{aligned}
$$

Thus, if we assume that $r_{k}^{(l+1)} \leq 2^{M} s_{k}^{(l)}$ for some fixed number $M$, we find that

$$
N_{k}\left(s_{k}^{(l)}, r_{k}^{(l+1)}\right) \leq \sum_{m=1}^{M} N_{k}\left(2^{m-1} s_{k}^{(l)}, 2^{m} s_{k}^{(l)}\right) \leq o(1),
$$

contradicting (32).

Similar to the blow-up analysis in [10], we then obtain the following result.

Proposition 2.3 There exist a subsequence $\left(u_{k}\right)$ and a constant $c^{(l+1)}$ such that

$$
\eta_{k}^{(l+1)}(x):=u_{k}\left(r_{k}^{(l+1)}\right)\left(u_{k}\left(r_{k}^{(l+1)} x\right)-u_{k}\left(r_{k}^{(l+1)}\right)\right) \rightarrow \eta_{0}^{(l+1)}(x)
$$

locally $C^{3}$-uniformly on $\mathbb{R}^{4} \backslash\{0\}$ as $k \rightarrow \infty$, where $\eta^{(l+1)}=\eta_{0}^{(l+1)}+c^{(l+1)} \in C^{\infty}\left(\mathbb{R}^{4}\right)$ solves equation (7) on $\mathbb{R}^{4}$ with

$$
\int_{\mathbb{R}^{4}} 6 e^{4 \eta^{(l+1)}} \mathrm{d} x=\Lambda_{1}
$$

Postponing the details of the proof of Proposition 2.3 to the next section, we now complete the proof of Theorem 1.2.

For a suitable subsequence $\left(u_{k}\right)$ Proposition 2.3 implies

$$
\lim _{L \rightarrow \infty} \lim _{k \rightarrow \infty} N_{k}\left(r_{k}^{(l+1)} / L, L r_{k}^{(l+1)}\right)=\Lambda_{1},
$$


and from (33) we obtain that

$$
\begin{aligned}
\lim _{L \rightarrow \infty} \lim _{k \rightarrow \infty} N_{k}\left(s_{k}^{(l)}, L r_{k}^{(l+1)}\right) & =\lim _{L \rightarrow \infty} \lim _{k \rightarrow \infty}\left(N_{k}\left(s_{k}^{(l)}, r_{k}^{(l+1)} / L\right)+N_{k}\left(r_{k}^{(l+1)} / L, L r_{k}^{(l+1)}\right)\right) \\
& =\Lambda_{1} .
\end{aligned}
$$

Our induction hypothesis (24) then yields

$$
\lim _{L \rightarrow \infty} \lim _{k \rightarrow \infty} \Lambda_{k}\left(L r_{k}^{(l+1)}\right)=\lim _{L \rightarrow \infty} \lim _{k \rightarrow \infty}\left(\Lambda_{k}\left(s_{k}^{(l)}\right)+N_{k}\left(s_{k}^{(l)}, L r_{k}^{(l+1)}\right)\right)=(l+1) \Lambda_{1} .
$$

Denote as

$$
w_{k}^{(l+1)}(x)=u_{k}\left(r_{k}^{(l+1)}\right)\left(u_{k}(x)-u_{k}\left(r_{k}^{(l+1)}\right)\right)
$$

the unscaled function $\eta_{k}^{(l+1)}$, satisfying the equation

$$
\Delta^{2} w_{k}^{(l+1)}=\lambda_{k} u_{k}\left(r_{k}^{(l+1)}\right) u_{k} e^{2 u_{k}^{2}}=: f_{k}^{(l+1)} \quad \text { in } \Omega=B_{R} .
$$

Observe that $f_{k}^{(l+1)} \geq e_{k}$ for $r \geq r_{k}^{(l+1)}$. We have the analogue of Lemma 2.1, which may be proved in the same fashion.

Lemma 2.4 For any $\varepsilon>0$, letting $T_{k}=T_{k}^{(l+1)}(\varepsilon)>0$ be such that $u_{k}\left(T_{k}\right)=\varepsilon u_{k}$ $\left(r_{k}^{(l+1)}\right) / 2$, for any constant $b<2$ and sufficiently large $k$ and $L$ there holds

$$
w_{k}^{(l+1)}(r) \leq b \log \left(\frac{r_{k}^{(l+1)}}{r}\right)+C \quad \text { on } B_{T_{k}} \backslash B_{L r_{k}^{(l+1)}}
$$

and we have

$$
\lim _{k \rightarrow \infty} N_{k}\left(s_{k}^{(l)}, T_{k}\right)=\Lambda_{1}
$$

For suitable numbers $s_{k}^{(l+1)}=T_{k}^{(l+1)}\left(\varepsilon_{k}\right)$ where $\varepsilon_{k} \downarrow 0$ as $k \rightarrow \infty$ then we have

$$
\lim _{k \rightarrow \infty} \Lambda_{k}\left(s_{k}^{(l+1)}\right)=(l+1) \Lambda_{1}
$$

and

$$
\lim _{L \rightarrow \infty} \lim _{k \rightarrow \infty}\left(\Lambda_{k}\left(s_{k}^{(l+1)}\right)-\Lambda_{k}\left(L r_{k}^{(l+1)}\right)\right)=\lim _{k \rightarrow \infty} \frac{r_{k}^{(l+1)}}{s_{k}^{(l+1)}}=\lim _{k \rightarrow \infty} \frac{u_{k}\left(s_{k}^{(l+1)}\right)}{u_{k}\left(r_{k}^{(l+1)}\right)}=0
$$

completing the induction step. In view of (3) and Lemma 2.2 the iteration must terminate after finitely many steps $1 \leq l \leq L$, after which

$$
N_{k}\left(s_{k}^{(L)}, R\right) \rightarrow 0 \quad \text { as } k \rightarrow \infty .
$$

This concludes the proof. 


\section{Proof of Proposition 2.3}

Throughout this section we let $r_{k}=r_{k}^{(l+1)}, s_{k}=s_{k}^{(l)}$, etc., and we set $r_{k}^{-}=r_{k}^{(l)}$. Note that monotonicity of $u_{k}$ and (26) give rise to the following estimate.

Lemma 3.1 There exists a constant $C$ independent of $k$ such that

$$
r^{2} u_{k}(r) \leq C r_{k}^{2} u_{k}\left(r_{k}\right) \quad \text { for all } r<r_{k} .
$$

Proof For $r \leq r_{k}$ we use (26) to estimate

$$
\frac{u_{k}^{2}(r) r^{4}}{u_{k}^{2}\left(r_{k}\right) r_{k}^{4}} P_{k}\left(r_{k}\right)=\omega_{3} \lambda_{k} r^{4} u_{k}^{2}(r) e^{2 u_{k}^{2}\left(r_{k}\right)} \leq P_{k}(r) \leq C_{0} N_{k}(r / 2, r) \leq C \Lambda .
$$

The claim follows from (34).

Denote as $v_{k}(x)=u_{k}\left(r_{k} x\right)$ the scaled function $u_{k}$. Also write $v_{k}(x)=v_{k}(r)$ for $r=|x|$. The following result is similar to Lemma 2.2 in [10].

Lemma 3.2 As $k \rightarrow \infty$ we have $v_{k}(1) \rightarrow \infty$ and there holds $v_{k}(x)-v_{k}(1) \rightarrow 0$ locally uniformly on $\left.\mathbb{R}^{4} \backslash\{0\}\right)$.

Proof Convergence $v_{k}(1)=u_{k}\left(r_{k}\right) \rightarrow \infty$ immediately follows from (34).

Let $\tilde{v}_{k}(x)=v_{k}(x)-v_{k}(1)$, satisfying the equation

$$
\Delta^{2} \tilde{v}_{k}=\lambda_{k} r_{k}^{4} v_{k} e^{2 v_{k}^{2}}=: g_{k} \geq 0 .
$$

We claim that $g_{k} \rightarrow 0$ locally uniformly away from 0 . Indeed, for $|x|=r \geq 1$ monotonicity implies that

$$
g_{k}(x) \leq \lambda_{k} r_{k}^{4} v_{k}(1) e^{2 v_{k}^{2}(1)}=\lambda_{k} r_{k}^{4} u_{k}\left(r_{k}\right) e^{2 u_{k}^{2}\left(r_{k}\right)}=C P_{k}\left(r_{k}\right) / u_{k}\left(r_{k}\right) \rightarrow 0
$$

as $k \rightarrow \infty$. On the other hand, for $1 / L \leq|x| \leq 1$, letting $t=\left|r_{k} x\right|$ from (26) we have the estimate

$$
g_{k}(x)=\lambda_{k} r_{k}^{4} u_{k}\left(r_{k} x\right) e^{2 u_{k}^{2}\left(r_{k} x\right)} \leq L^{4} \lambda_{k} t^{4} u_{k}(t) e^{2 u_{k}^{2}(t)}=C L^{4} P_{k}(t) / u_{k}(t) \rightarrow 0 .
$$

Since from (4) we also have the uniform $L^{2}$-bound

$$
\left\|\nabla^{2} \tilde{v}_{k}\right\|_{L^{2}}=\left\|\nabla^{2} u_{k}\right\|_{L^{2}} \leq C,
$$

we may extract a subsequence $\left(u_{k}\right)$ such that $\Delta \tilde{v}_{k}=: \tilde{w}_{k} \rightarrow \tilde{w}$ weakly in $L_{l o c}^{2}\left(\mathbb{R}^{4}\right)$ and in $C_{l o c}^{1, \alpha}\left(\mathbb{R}^{4} \backslash\{0\}\right)$, where $\tilde{w}$ is harmonic away from the origin. In addition, $\tilde{w} \in L^{2}\left(\mathbb{R}^{4}\right)$; since the point $x=0$ has vanishing $H^{2}$-capacity, we then have $\Delta \tilde{w}=0$ in the distribution sense on all of $\mathbb{R}^{4}$ and $\tilde{w}$ is a smooth, everywhere harmonic function. Again invoking the fact that $\tilde{w} \in L^{2}\left(\mathbb{R}^{4}\right)$, then we see that $\tilde{w}$ vanishes identically; that is, we have $\Delta \tilde{v}_{k} \rightarrow 0$ weakly in $L_{\text {loc }}^{2}\left(\mathbb{R}^{4}\right)$ and in $C_{\text {loc }}^{1, \alpha}\left(\mathbb{R}^{4} \backslash\{0\}\right)$.

The $L^{2}$-bound (43) together with the fact that $\tilde{v}_{k}(1)=0$ by the $H^{2}$-estimates for the Laplace operator also yields weak $H^{2}$-convergence $\tilde{v}_{k} \rightarrow \tilde{v}$ in $B_{1}$ and then, by a variant of the Poincaré inequality, also local weak $H^{2}$-convergence on all of $\mathbb{R}^{4}$, where $\tilde{v}$ is smooth and harmonic. Since $\tilde{v}(1)=0$, moreover, by the maximum principle $\tilde{v}$ vanishes throughout $B_{1}$. By the unique continuation property of harmonic functions then $\tilde{v}$ vanishes everywhere. Recalling that for radially symmetric functions weak $H^{2}$-convergence implies locally uniform convergence away from the origin, we obtain the claim. 
From the definition

$$
\eta_{k}(x)=u_{k}\left(r_{k}\right)\left(u_{k}\left(r_{k} x\right)-u_{k}\left(r_{k}\right)\right)=v_{k}(1)\left(v_{k}(x)-v_{k}(1)\right),
$$

with $r_{k}=r_{k}^{(l+1)}$, etc., and with $\hat{v}_{k}=v_{k} / v_{k}(1) \rightarrow 1$ locally uniformly on $\left.\mathbb{R}^{4} \backslash\{0\}\right)$ by Lemma 3.2, we derive the equation

$$
\begin{aligned}
\Delta^{2} \eta_{k} & =r_{k}^{4} u_{k}\left(r_{k}\right)\left(\Delta^{2} u_{k}\right)\left(r_{k} \cdot\right)=\lambda_{k} r_{k}^{4} u_{k}^{2}\left(r_{k}\right) e^{2 u_{k}^{2}\left(r_{k}\right)} \hat{v}_{k} e^{2\left(v_{k}^{2}-v_{k}^{2}(1)\right)} \\
& =\mu_{k} \hat{v}_{k} e^{2 \eta_{k}\left(\hat{v}_{k}+1\right)}
\end{aligned}
$$

where $\mu_{k}=\omega_{3}^{-1} P_{k}\left(r_{k}\right) \geq c_{0}>0$ by (34). Note that by monotonicity of $u_{k}$ we have

$$
\eta_{k} \leq 0, \hat{v}_{k} \leq 1,0 \leq \Delta^{2} \eta_{k} \leq \mu_{k} \leq C \Lambda \text { outside } B_{1}(0),
$$

whereas $\eta_{k} \geq 0, \hat{v}_{k} \geq 1$ in $B_{1}(0)$. Thus, monotonicity of $u_{k}$ together with (26) yield

$$
0 \leq \mu_{k} e^{4 \eta_{k}(x)} \leq \Delta^{2} \eta_{k}(x) \leq r_{k}^{4} e_{k}\left(r_{k} x\right)=|x|^{-4} \omega_{3}^{-1} P_{k}\left(r_{k}|x|\right) \leq C|x|^{-4}
$$

for any $x \in B_{1}(0)$; moreover, for any $L \geq 1$ and sufficiently large $k$ by (3) we have

$$
\int_{B_{1}(0)} \Delta^{2} \eta_{k} \mathrm{~d} x \leq \int_{B_{L}(0)} \hat{v}_{k} \Delta^{2} \eta_{k} \mathrm{~d} x=\int_{B_{L r_{k}}(0)} u_{k} \Delta^{2} u_{k} \mathrm{~d} x \leq C \Lambda .
$$

Finally, in view of (25) and (33) there holds

$$
\begin{aligned}
\int_{B_{1 / L}(0)} \Delta^{2} \eta_{k} \mathrm{~d} x & =\int_{B_{r_{k} / L}(0)} u_{k}\left(r_{k}\right) \Delta^{2} u_{k} \mathrm{~d} x \\
& \leq \int_{B_{r_{k} / L} \backslash B_{L r_{k}^{-}}(0)} u_{k} \Delta^{2} u_{k} \mathrm{~d} x+\frac{u_{k}\left(r_{k}\right)}{u_{k}\left(L r_{k}^{-}\right)} \int_{B_{L r_{k}^{-}}(0)} u_{k} \Delta^{2} u_{k} \mathrm{~d} x \\
& \leq N_{k}\left(L r_{k}^{-}, r_{k} / L\right)+C \Lambda \frac{u_{k}\left(s_{k}\right)}{u_{k}\left(L r_{k}^{-}\right)} \rightarrow 0,
\end{aligned}
$$

if we first let $k \rightarrow \infty$ and then pass to the limit $L \rightarrow \infty$.

Recall that

$$
v_{k}:=-\Delta \eta_{k}=-v_{k}(1) \Delta v_{k} \geq 0
$$

in view of (2) and the maximum principle. The following result is similar to [10], estimate (21).

Lemma 3.3 For any $K>3$ there exists a constant $C(K)$ such that

$$
\int_{B_{L}(0)} v_{k} \mathrm{~d} x \leq C(K) L^{2}+C L^{4} \frac{1+\log K}{1+K^{2}}
$$

for any $L>1$ and any sufficiently large $k$.

Proof Extend $v_{k}$ to $v_{k} \in H_{0}^{2}\left(\mathbb{R}^{4}\right)$ by letting

$$
v_{k}(x)=-\tau\left(r_{k} x\right) v_{k}\left(R_{k}^{2} x /|x|^{2}\right) \quad \text { for }|x|>R_{k}=R / r_{k},
$$


with a fixed cut-off function $\tau \in C_{0}^{\infty}\left(B_{2 R}(0)\right)$ such that $\tau \equiv 1$ in a neighborhood of $B_{R}(0)$. Observe that we have the uniform bound

$$
\left\|\nabla v_{k}\right\|_{L^{4}} \leq C\left\|\nabla^{2} v_{k}\right\|_{L^{2}} \leq C\left\|\nabla^{2} u_{k}\right\|_{L^{2}} \leq C \Lambda .
$$

For any $y \in \mathbb{R}^{4}$ and any $r>0$ define

$$
c_{k}^{(y, r)}=f_{B_{r}(y)} v_{k} \mathrm{~d} x,
$$

where we denote as $f_{A}=\frac{1}{\operatorname{Vol}(A)} \int_{A}$ the mean value on a domain $A \subset \mathbb{R}^{4}$. Similar to [2], Lemma 4.2, the Poincaré inequality (or the embedding $W^{1,4}\left(\mathbb{R}^{4}\right) \rightarrow B M O\left(\mathbb{R}^{4}\right)$ ) together with Lemma 3.2 yields the uniform estimate

$$
\left.\left|c_{k}^{(y, 1)}-v_{k}(1)\right| \leq \mid c_{k}^{(y, 1)}-c_{k}^{(1,1 / 2)}\right)|+| c_{k}^{(1,1 / 2)}-v_{k}(1) \mid \leq C \log (1+|y|)+C
$$

for all $y \in \mathbb{R}^{4}$ and sufficiently large $k$. The proof of [10], estimate (21), now may be carried over essentially unchanged to the present situation to yield our claim. The only modification required is replacing [10], estimate (19), by the estimate

$$
\begin{aligned}
\int_{|y| \leq K} \lambda_{k} r_{k}^{4} u_{k}\left(r_{k}\right) v_{k}(y) e^{2 v_{k}^{2}(y)} \mathrm{d} y= & \int_{|y| \leq 1} \cdots+\int_{1 \leq|y| \leq K} \cdots \\
& \leq \int_{|y| \leq 1} \lambda_{k} r_{k}^{4} v_{k}^{2}(y) e^{2 v_{k}^{2}(y)} \mathrm{d} y \\
& +C K^{4} \lambda_{k} r_{k}^{4} u_{k}^{2}\left(r_{k}\right) e^{2 u_{k}^{2}\left(r_{k}\right)} \leq C\left(1+K^{4}\right),
\end{aligned}
$$

which follows from (3), (26), and monotonicity of $u_{k}$.

Lemma 3.4 There exist a subsequence $\left(u_{k}\right)$ such that $v_{k} \rightarrow v$ locally uniformly in $C^{1, \alpha}$ on $\mathbb{R}^{4} \backslash\{0\}$ as $k \rightarrow \infty$.

Proof For any $L>1$ decompose $v_{k}=H_{k}+N_{k}$ on $B_{L} \backslash B_{1 / L}(0)$, where $\Delta H_{k}=0$ in $B_{L} \backslash B_{1 / L}(0)$, and where $N_{k}=0$ on $\partial\left(B_{L} \backslash B_{1 / L}(0)\right)$. After passing to a further subsequence, if necessary, in view of (45), (46) we may assume that $N_{k} \rightarrow N$ in $C^{1, \alpha}$ on $B_{L} \backslash B_{1 / L}(0)$ as $k \rightarrow \infty$. On the other hand, by the mean value property of harmonic functions and Lemma 3.3 together with the fact that $v_{k} \geq 0$ a subsequence $H_{k} \rightarrow H$ locally $C^{2}$-uniformly on $B_{L} \backslash B_{1 / L}(0)$ as $k \rightarrow \infty$, and the proof is complete.

Lemma 3.5 There exist a subsequence $\left(u_{k}\right)$ such that $\eta_{k} \rightarrow \eta_{0}$ locally uniformly in $C^{3, \alpha}$ on $\mathbb{R}^{4} \backslash\{0\}$ as $k \rightarrow \infty$.

Proof Similar to the proof of Lemma 3.4 for any $L>1$ we decompose $\eta_{k}=h_{k}+l_{k}$ on $B_{L} \backslash B_{1 / L}(0)$, where $\Delta h_{k}=0$ in $B_{L} \backslash B_{1 / L}(0)$, and where $l_{k}=0$ on $\partial\left(B_{L} \backslash B_{1 / L}(0)\right)$. By Lemma 3.4 we may assume that $l_{k} \rightarrow l$ in $C^{3, \alpha}$ on $B_{L} \backslash B_{1 / L}(0)$ as $k \rightarrow \infty$. Taking account of the estimates $\eta_{k}(r) \geq \eta_{k}(1)=0$ for $1 / L<r<1$ and $\eta_{k}(r) \leq \eta_{k}(1)=0$ for $1<r<L$, respectively, from Harnack's inequality we conclude that either a subsequence $h_{k} \rightarrow h$ locally $C^{4}$-uniformly on $B_{L} \backslash B_{1 / L}(0)$, or $h_{k} \rightarrow \infty$ and hence $\eta_{k} \rightarrow \infty$ locally uniformly on $B_{1} \backslash B_{1 / L}(0)$ as $k \rightarrow \infty$. But the latter possibility is excluded by (46), and the assertion follows. 
Proof of Proposition 2.3 Since $\Delta^{2} \eta_{k} \geq 0$ by (47) is uniformly bounded in $L^{1}\left(B_{L}(0)\right)$, from Lemma 3.5 and elliptic regularity we also obtain that the sequence $\left(\eta_{k}\right)$ is bounded in $W^{2, q}\left(B_{L}(0)\right)$ for any $q<2$ and any $L \geq 1$. In addition to the assertion of Lemma 3.5 we thus may assume that $\eta_{k} \rightarrow \eta_{0}$ weakly locally in $W^{2, q}$ as $k \rightarrow \infty$.

By Lemmas 3.2 and 3.5 we may pass to the limit $k \rightarrow \infty$ in Eq. (44) to see that $\eta_{0}$ solves the equation

$$
\Delta^{2} \eta_{0}=\mu_{0} e^{4 \eta_{0}} \quad \text { on } \mathbb{R}^{4} \backslash\{0\},
$$

for some constant $\mu_{0}=\lim _{k \rightarrow \infty} \mu_{k}>0$. Moreover, from Lemma 3.3 we obtain that

$$
\lim _{L \rightarrow \infty}\left(L^{-4} \int_{B_{L}(0)} \Delta \eta_{0} \mathrm{~d} x\right)=0 .
$$

In addition, by Fatou's lemma, Lemma 3.2, and (47), for any $L>1$ with a uniform constant $C$ we have

$$
\begin{aligned}
\int_{B_{L} \backslash B_{1 / L}(0)} e^{4 \eta_{0}} \mathrm{~d} x & \leq \liminf _{k \rightarrow \infty} \int_{B_{L} \backslash B_{1 / L}(0)} \hat{v}_{k}^{2} e^{\left.2 \eta_{k}\left(\hat{v}_{k}(x)+1\right)\right)} \mathrm{d} x \\
& =\liminf _{k \rightarrow \infty} \int_{B_{L} \backslash B_{1 / L}(0)} \mu_{k}^{-1} \hat{v}_{k} \Delta^{2} \eta_{k} \mathrm{~d} x \leq C \Lambda .
\end{aligned}
$$

Passing to the limit $L \rightarrow \infty$, we see that $e^{4 \eta_{0}} \in L^{1}\left(\mathbb{R}^{4}\right)$. Since in view of (48) we also have

$$
\int_{B_{1 / L}(0)} \Delta^{2} \eta_{0} \mathrm{~d} x \leq \limsup _{k \rightarrow \infty} \int_{B_{1 / L}(0)} \Delta^{2} \eta_{k} \mathrm{~d} x \rightarrow 0
$$

as $L \rightarrow \infty$, it follows that $\eta_{0}$ extends to a distribution solution of (50) on all of $\mathbb{R}^{4}$. Writing $\mu_{0}=6 e^{4 c}$ for some constant $c=c^{(l+1)} \in \mathbb{R}$, and letting $\eta=\eta_{0}+c$ we find that $\eta$ solves Eq. (7). The desired characterization of $\eta$ then can be obtained exactly as in [10], Proposition 2.4, from the bound (51) and Lin's [6] classification of all solutions to equation (7) on $\mathbb{R}^{4}$ with $e^{4 \eta} \in L^{1}\left(\mathbb{R}^{4}\right)$.

\section{The general case}

For the proof of Theorem 1.2 in the general case fix an index $1 \leq i \leq I$ and let $x_{k}=x_{k}^{(i)} \rightarrow x^{(i)}, 0<r_{k}=r_{k}^{(i)} \rightarrow 0$ be determined as in Theorem 1.1 so that $u_{k}\left(x_{k}\right)=\max _{\left|x-x_{k}\right| \leq L r_{k}} u_{k}(x)$ for any $L>0$ and sufficiently large $k$ and such that

$$
\eta_{k}(x)=\eta_{k}^{(i)}(x):=u_{k}\left(x_{k}\right)\left(u_{k}\left(x_{k}+r_{k} x\right)-u_{k}\left(x_{k}\right)\right) \rightarrow \log \left(\frac{1}{1+|x|^{2}}\right)
$$

as $k \rightarrow \infty$. For each $k$ we may shift the origin so that henceforth we may assume that $x_{k}=0$ for all $k$. Denote as $\Omega_{k}=\Omega_{k}^{(i)}$ the shifted domain $\Omega$. We also extend $u_{k}$ to $u_{k} \in W_{0}^{2,2}\left(\mathbb{R}^{4}\right)$, still satisfying the uniform bound (4) up to a constant. Again we let $e_{k}=\lambda_{k} u_{k}^{2} e^{2 u_{k}^{2}}, f_{k}=\lambda_{k} u_{k}(0) u_{k} e^{2 u_{k}^{2}}$, and for $0<r<R$ we set

型 Springer 


$$
\Lambda_{k}(r)=\int_{B_{r}} e_{k} \mathrm{~d} x, \quad \sigma_{k}(r)=\int_{B_{r}} f_{k} \mathrm{~d} x
$$

satisfying Eq. (17).

Also introduce the spherical mean $\bar{u}_{k}(r)=f_{\partial B_{r}} u_{k}$ do of $u_{k}$ on $\partial B_{r}$, and so on, and set $\tilde{e}_{k}=\lambda_{k} \bar{u}_{k}^{2} e^{2 \bar{u}_{k}^{2}}$. Recall that in view of (2) and the maximum principle we have $\Delta u_{k} \leq 0$ in $\Omega_{k}$ and then also

$$
\bar{u}_{k}^{\prime}(r)=f_{\partial B_{r}} \frac{x}{|x|} \cdot \nabla u_{k} \mathrm{~d} o=\frac{r}{4} f_{B_{r}} \Delta u_{k} \mathrm{~d} x \leq 0 ;
$$

that is, $\bar{u}_{k}(r)$ is non-increasing in $r$ as long as $B_{r} \subset \Omega_{k}$.

The spherical mean $\bar{w}_{k}$ of the function

$$
w_{k}(x)=u_{k}(0)\left(u_{k}(x)-u_{k}(0)\right),
$$

satisfies the equation

$$
\Delta^{2} \bar{w}_{k}=\lambda_{k} u_{k}(0) \overline{u_{k} e^{2 u_{k}^{2}}}=\bar{f}_{k}
$$

Note that by Jensen's inequality we have

$$
\tilde{e}_{k} \leq \bar{e}_{k}
$$

hence

$$
\tilde{\Lambda}_{k}(r):=\int_{B_{r}} \tilde{e}_{k} \mathrm{~d} x \leq \Lambda_{k}(r), \quad \int_{B_{r}} \bar{f}_{k} \mathrm{~d} x=\sigma_{k}(r) .
$$

Observe that in analogy with (17) Theorem 1.1 implies

$$
\lim _{L \rightarrow \infty} \lim _{k \rightarrow \infty} \tilde{\Lambda}_{k}\left(L r_{k}\right)=\lim _{L \rightarrow \infty} \lim _{k \rightarrow \infty} \Lambda_{k}\left(L r_{k}\right)=\lim _{L \rightarrow \infty} \lim _{k \rightarrow \infty} \sigma_{k}\left(L r_{k}\right)=\Lambda_{1} .
$$

To proceed, we also need the following gradient estimate similar to Druet [4], Proposition 2 . For any $k \in \mathbb{N}, x \in \Omega$ we let

$$
R_{k}(x)=\inf _{1 \leq j \leq I}\left|x-x_{k}^{(j)}\right| .
$$

Proposition 4.1 There exists a uniform constant $C$ such that for all $x \in \Omega$ there holds

$$
R_{k}(x) u_{k}(x)\left|\nabla u_{k}(x)\right| \leq C,
$$

uniformly in $k \in \mathbb{N}$.

The proof of Proposition 4.1 is given in the next section.

Recalling that $x_{k}^{(i)}=0$, we let

$$
\rho_{k}=\rho_{k}^{(i)}=\frac{1}{2} \inf _{j \neq i}\left|x_{k}^{(j)}\right|
$$

and we set $\rho_{k}=\infty$ if $\{j ; j \neq i\}=\emptyset$, that is, if there is no other concentration point but $x_{k}^{(i)}$. We now use Proposition 4.1 to deal with concentrations around the point $x_{k}^{(i)}$ 
at scales which are small with respect to $\rho_{k}$. Indeed, for $|x| \leq \rho_{k}$ we have $|x|=R_{k}(x)$; therefore, by Proposition 4.1 for any $0<r \leq \rho_{k}$ with a uniform constant $C$ there holds

$$
0 \leq \sup _{x \in \partial B_{r}} u_{k}^{2}(x)-\inf _{x \in \partial B_{r}} u_{k}^{2}(x) \leq C r \sup _{x \in \partial B_{r}}\left|\nabla u_{k}^{2}(x)\right| \leq C .
$$

Hence

$$
\sup _{x \in \partial B_{r}} e^{2 u_{k}^{2}(x)} \leq C e^{2 \bar{u}_{k}^{2}(r)},
$$

and we conclude the estimate

$$
\sup _{x \in \partial B_{r}} u_{k}^{2}(x) e^{2 u_{k}^{2}(x)} \leq C_{3}\left(1+\bar{u}_{k}^{2}(r)\right) e^{2 \bar{u}_{k}^{2}(r)}
$$

with a uniform constant $C_{3}$.

Also note that in view of (9) and our choice of $x_{k}^{(i)}$ there holds $u_{k}(x) \leq u_{k}(0)$ for all $|x| \leq \rho_{k}$; hence at this scale there also holds the inequality $e_{k} \leq f_{k}$.

Similarly to Lemma 2.1 then we obtain

Lemma 4.2 For any $\varepsilon>0$, if there is $0<T_{k} \leq \rho_{k}$ such that $\bar{u}_{k}\left(T_{k}\right)=\varepsilon u_{k}(0) / 2$, then for any constant $b<2$ and sufficiently large $k$ there holds

$$
\bar{w}_{k}(r) \leq b \log \left(\frac{r_{k}}{r}\right)+C \quad \text { for } 0 \leq r \leq T_{k}
$$

and we have

$$
\lim _{k \rightarrow \infty} \tilde{\Lambda}_{k}\left(T_{k}\right)=\lim _{k \rightarrow \infty} \sigma_{k}\left(T_{k}\right)=\Lambda_{1}
$$

Proof First observe that (2) together with (57) and the fact that $u_{k}(0) \rightarrow \infty$ as $k \rightarrow \infty$ implies that $\operatorname{dist}\left(0, \partial \Omega_{k}\right) \geq T_{k}$ for sufficiently large $k$. Hence for such $k$ the spherical averages $\bar{u}_{k}(r)$ are well-defined for any $r \leq T_{k}$.

As in the proof of Lemma 2.1 for $t=t_{k} \leq T_{k}$ decompose $\bar{w}_{k}=g_{k}+h_{k}$ on $B_{t}$, where $g_{k}$ satisfies $g_{k}=\partial_{\nu} g_{k}=0$ on $\partial B_{t}$ and where $h_{k}(r)=\bar{w}_{k}(t)+d_{k}\left(r^{2}-t^{2}\right)$ satisfies $\Delta^{2} h_{k}=0$. Then as before we derive the identity

$$
\Delta \bar{w}_{k}-4 t^{-1} \partial_{\nu} \bar{w}_{k}=\Delta g_{k} \quad \text { on } \partial B_{t}
$$

and for any $b<2$, any $t=t_{k} \geq L r_{k}$, for sufficiently large $L$ and $k$ from (20) and (56) we obtain

$$
\Delta g_{k} \geq \frac{2 b}{t^{2}} \quad \text { on } \partial B_{t}
$$

Again it follows that the expression $\frac{\bar{w}_{k}^{\prime}}{r}+\frac{b}{r^{2}}$ is non-decreasing for $r \geq L r_{k}$ when $L>0$ and $k$ are chosen sufficiently large. Since $\bar{w}_{k}^{\prime}=u_{k}(0) \bar{u}_{k}^{\prime} \leq 0$ on $B_{T_{k}}$ we may then deduce (60) in the same way as (18) from (21).

Using (14) and (58), for $L r_{k} \leq r \leq T_{k} \leq \rho_{k}$ with sufficiently large $L>0$ and $k$ from (5) and (60) then we obtain

$$
\begin{aligned}
\bar{f}_{k} & \leq C \lambda_{k} u_{k}^{2}(0) e^{2 u_{k}^{2}(0)} e^{4\left(1+\frac{\bar{w}_{k}}{2 u_{k}^{2}(0)}\right) \bar{w}_{k}} \\
& \leq C \lambda_{k} r_{k}^{4} u_{k}^{2}(0) e^{2 u_{k}^{2}(0)} r_{k}^{-4} e^{(2+\varepsilon) \bar{w}_{k}} \leq C r_{k}^{-4}\left(\frac{r_{k}}{r}\right)^{(2+\varepsilon) b},
\end{aligned}
$$

and we complete the proof as before. 
With the help of Proposition 4.1 we can improve the estimate (61) to obtain the analogue of (19). For $0 \leq s<t \leq \rho_{k}$ we let

$$
N_{k}(s, t)=\int_{B_{t} \backslash B_{s}} e_{k} \mathrm{~d} x=\int_{B_{t} \backslash B_{s}} \lambda_{k} u_{k}^{2} e^{2 u_{k}^{2}} \mathrm{~d} x,
$$

as before and define

$$
\tilde{N}_{k}(s, t)=\int_{B_{t} \backslash B_{s}} \tilde{e}_{k} \mathrm{~d} x=\omega_{3} \int_{s}^{t} \lambda_{k} r^{3} \bar{u}_{k}^{2} e^{2 \bar{u}_{k}^{2}} \mathrm{~d} r \leq N_{k}(s, t) .
$$

Also let

$$
P_{k}(t)=t \frac{\partial}{\partial t} N_{k}(s, t)=t \int_{\partial B_{t}} e_{k} \mathrm{~d} o
$$

and set

$$
\tilde{P}_{k}(t)=t \frac{\partial}{\partial t} \tilde{N}_{k}(s, t)=t \int_{\partial B_{t}} \tilde{e}_{k} \mathrm{~d} o=\omega_{3} \lambda_{k} t^{4} \bar{u}_{k}^{2}(t) e^{2 \bar{u}_{k}^{2}(t)} \leq P_{k}(t) .
$$

The analogue of (26) then holds for $\tilde{N}_{k}$ and $\tilde{P}_{k}$. Also note that (59) implies the estimate

$$
N_{k}(s, t) \leq C_{3} \tilde{N}_{k}(s, t)+o(1), \quad P_{k}(t) \leq C_{3} \tilde{P}_{k}(t)+o(1),
$$

with error $o(1) \rightarrow 0$ as $k \rightarrow \infty$, uniformly in $s \leq t \leq \rho_{k}$.

Lemma 4.3 For any $\varepsilon>0$, if $T_{k}=T_{k}(\varepsilon) \leq \rho_{k}$ is as in Lemma 4.2, then we have

$$
\lim _{k \rightarrow \infty} \Lambda_{k}\left(T_{k}\right)=\Lambda_{1}
$$

Proof In view of (56) and Lemma 4.2 we have

$$
\lim _{L \rightarrow \infty} \lim _{k \rightarrow \infty} \tilde{N}_{k}\left(L r_{k}, T_{k}\right) \leq \lim _{k \rightarrow \infty} \tilde{\Lambda}_{k}\left(T_{k}\right)-\lim _{L \rightarrow \infty} \lim _{k \rightarrow \infty} \tilde{\Lambda}_{k}\left(L r_{k}\right)=0 .
$$

Since by (63) with error $o(1) \rightarrow 0$ as $k \rightarrow \infty$ we can estimate

$$
\Lambda_{k}\left(L r_{k}\right) \leq \Lambda_{k}\left(T_{k}\right)=\Lambda_{k}\left(L r_{k}\right)+N_{k}\left(L r_{k}, T_{k}\right) \leq \Lambda_{k}\left(L r_{k}\right)+C_{3} \tilde{N}_{k}\left(L r_{k}, T_{k}\right)+o(1),
$$

upon letting $k \rightarrow \infty$ and then passing to the limit $L \rightarrow \infty$, from (56) we obtain the claim.

Under the assumptions of Lemma 4.2, as in the radially symmetric case for a suitable sequence $\varepsilon_{k} \downarrow 0$ as $k \rightarrow \infty$ and corresponding numbers $s_{k}=T_{k}\left(\varepsilon_{k}\right) \leq \rho_{k}$ now we have

$$
\lim _{k \rightarrow \infty} \Lambda_{k}\left(s_{k}\right)=\Lambda_{1}
$$

and

$$
\lim _{L \rightarrow \infty} \lim _{k \rightarrow \infty}\left(\Lambda_{k}\left(s_{k}\right)-\Lambda_{k}\left(L r_{k}\right)\right)=\lim _{k \rightarrow \infty} \frac{\bar{u}_{k}\left(s_{k}\right)}{\bar{u}_{k}\left(r_{k}\right)}=\lim _{k \rightarrow \infty} \frac{r_{k}}{s_{k}}=0 .
$$


By slight abuse of notation we let $r_{k}=r_{k}^{(1)}, s_{k}=s_{k}^{(1)}$ and proceed by iteration as before. Suppose that for some integer $l \geq 1$ we already have determined numbers $r_{k}^{(1)}<s_{k}^{(1)}<\cdots<r_{k}^{(l)}<s_{k}^{(l)} \leq \rho_{k}$ such that

$$
\lim _{k \rightarrow \infty} \Lambda_{k}\left(s_{k}^{(l)}\right)=l \Lambda_{1}
$$

and

$$
\lim _{L \rightarrow \infty} \lim _{k \rightarrow \infty}\left(\Lambda_{k}\left(s_{k}^{(l)}\right)-\Lambda_{k}\left(L r_{k}^{(l)}\right)\right)=\lim _{k \rightarrow \infty} \frac{u_{k}\left(s_{k}^{(l)}\right)}{u_{k}\left(r_{k}^{(l)}\right)}=\lim _{k \rightarrow \infty} \frac{r_{k}^{(l)}}{s_{k}^{(l)}}=0 .
$$

Similar to Lemma 2.2 then we have the following result.

\section{Lemma 4.4}

(i) Suppose that for some $s_{k}^{(l)}<t_{k} \leq \rho_{k}$ there holds

$$
\sup _{s_{k}^{(l)}<t<t_{k}} P_{k}(t) \rightarrow 0 \quad \text { as } k \rightarrow \infty \text {. }
$$

Then we have

$$
\lim _{k \rightarrow \infty} N_{k}\left(s_{k}^{(l)}, t_{k}\right)=0
$$

(ii) Conversely, if for some $t_{k}>s_{k}^{(l)}$ and a subsequence $\left(u_{k}\right)$ there holds

$$
\lim _{k \rightarrow \infty} N_{k}\left(s_{k}^{(l)}, t_{k}\right)=c_{0}>0, \lim _{k \rightarrow \infty} t_{k} / \rho_{k}=0
$$

then either $c_{0} \geq 2 \omega_{3}$, or we have

$$
\liminf _{k \rightarrow \infty} P_{k}\left(t_{k}\right) \geq 2 c_{0}
$$

and

$$
\lim _{L \rightarrow \infty} \liminf _{k \rightarrow \infty} N_{k}\left(s_{k}^{(l)}, L t_{k}\right) \geq 2 \omega_{3}, \quad \lim _{L \rightarrow \infty} \limsup _{k \rightarrow \infty} N_{k}\left(s_{k}^{(l)}, t_{k} / L\right)=0
$$

Proof (i) In view of (63) we may consider $\tilde{N}_{k}(s, t)$ and $\tilde{P}_{k}(t)$ instead of $N_{k}(s, t)$ and $P_{k}(t)$. For $s=s_{k}^{(l)}<t$ similar to (27) we integrate by parts to obtain

$$
\begin{aligned}
4 \tilde{N}_{k}(s, t) & =\left.\tilde{P}_{k}(r)\right|_{s} ^{t}-\int_{B_{t} \backslash B_{s}} x \cdot \nabla \tilde{e}_{k} \mathrm{~d} x \\
& \leq \tilde{P}_{k}(t)-4 \int_{B_{t} \backslash B_{s}} \frac{\bar{u}_{k}}{u_{k}(0)} r \bar{w}_{k}^{\prime} \tilde{e}_{k} \mathrm{~d} x+o(1),
\end{aligned}
$$

where $r \bar{w}_{k}^{\prime}=x \cdot \nabla \bar{w}_{k}$, as usual, and with error $o(1) \rightarrow 0$ as $k \rightarrow \infty$, uniformly in $s \leq t$. As in the proof of Lemma 2.2 then for sufficiently large $L$ and $k$ we obtain the estimate

$$
r \bar{w}_{k}^{\prime}(r) \geq-\frac{\int_{0}^{r} r^{\prime} \sigma_{k}\left(r^{\prime}\right) \mathrm{d} r^{\prime}}{2 \omega_{3} r^{2}} \geq-\frac{\sigma_{k}(r)}{4 \omega_{3}} \quad \text { for all } r \geq L r_{k}
$$


analogous to (28) and we conclude that

$$
-\frac{4 \bar{u}_{k}}{u_{k}(0)} r \bar{w}_{k}^{\prime} \leq \omega_{3}^{-1} N_{k}(s, r)+o(1) .
$$

It follows that

$$
4 \tilde{N}_{k}(s, t) \leq \tilde{P}_{k}(t)+\omega_{3}^{-1} N_{k}(s, r) \tilde{N}_{k}(s, t)+o(1) .
$$

Thus, if we assume that

$$
\sup _{s<t<t_{k}} \tilde{P}_{k}(t) \leq \sup _{s<t<t_{k}} P_{k}(t) \rightarrow 0 \quad \text { as } k \rightarrow \infty,
$$

upon letting $t$ increase from $t=s_{k}^{(l)}$ to $t_{k}$ as before we find

$$
\lim _{k \rightarrow \infty} N_{k}\left(s_{k}^{(l)}, t_{k}\right) \leq C_{3} \lim _{k \rightarrow \infty} \tilde{N}_{k}\left(s_{k}^{(l)}, t_{k}\right)=0,
$$

as claimed.

(ii) On account of (62), (63), the second assertion follows from (66) exactly as in the proof of Lemma 2.2.

Continuing to argue as in the radial case, if for some $s_{k}^{(l)}<t_{k} \leq \rho_{k}$ there holds

$$
\limsup _{k \rightarrow \infty} N_{k}\left(s_{k}^{(l)}, t_{k}\right)>0, \quad \lim _{k \rightarrow \infty} t_{k} / \rho_{k}=0,
$$

from Lemma 4.4 we infer that for a subsequence $\left(u_{k}\right)$ and suitable numbers $r_{k}^{(l+1)} \in$ ]$s_{k}^{(l)}, t_{k}[$ we have

$$
0<\lim _{k \rightarrow \infty} N_{k}\left(s_{k}^{(l)}, r_{k}^{(l+1)}\right)=c_{0}<2 \omega_{3}, \quad \liminf _{k \rightarrow \infty} P_{k}\left(r_{k}^{(l+1)}\right)>0,
$$

while

$$
\lim _{L \rightarrow \infty} \limsup _{k \rightarrow \infty} N_{k}\left(s_{k}^{(l)}, r_{k}^{(l+1)} / L\right)=0
$$

Note that as in the radial case we have $r_{k}^{(l+1)} / s_{k}^{(l)} \rightarrow \infty$ as $k \rightarrow \infty$ by (26), (62), and (65); in addition, there holds

$$
\lim _{k \rightarrow \infty} r_{k}^{(l+1)} / \rho_{k} \leq \lim _{k \rightarrow \infty} t_{k} / \rho_{k}=0 .
$$

Moreover, we find the analogue of Proposition 2.3.

Proposition 4.5 There exist a subsequence $\left(u_{k}\right)$ and a constant $c^{(l+1)}$ such that

$$
\eta_{k}^{(l+1)}(x):=\bar{u}_{k}\left(r_{k}^{(l+1)}\right)\left(u_{k}\left(r_{k}^{(l+1)} x\right)-\bar{u}_{k}\left(r_{k}^{(l+1)}\right)\right) \rightarrow \eta_{0}^{(l+1)}(x)
$$

locally $C^{3}$-uniformly on $\mathbb{R}^{4} \backslash\{0\}$ as $k \rightarrow \infty$, where $\eta^{(l+1)}=\eta_{0}^{(l+1)}+c^{(l+1)} \in C^{\infty}\left(\mathbb{R}^{4}\right)$ solves equation (7) on $\mathbb{R}^{4}$ with

$$
\int_{\mathbb{R}^{4}} 6 e^{4 \eta^{(l+1)}} \mathrm{d} x=\Lambda_{1} .
$$


Proposition 4.5 is a special case of Proposition 4.7, whose proof will be presented in Sect. 6.

From Proposition 4.5 the energy estimate at the scale $r_{k}^{(l+1)}$ follows as in the radial case. Likewise, under assumptions similar to Lemma 4.2 we obtain the analogue of Lemma 2.4. By iteration, as in the radial case, we determine the concentration profiles and concentration energies for secondary concentrations around the point $x_{k}^{(i)}=0$ at scales up to some $s_{k}^{\left(l_{0}\right)}<r_{k}^{\left(l_{0}+1\right)}$ satisfying (68) and such that (64), (65) are valid for all $l$ up to some maximal index $l_{0} \geq 0$. (We set $s_{k}^{(0)}=0$.)

If $\rho_{k}=\infty$, moreover, for any $\varepsilon>0$ we can find numbers $0<T_{k}=T_{k}(\varepsilon)<\rho_{k}$ such that $\bar{u}_{k}\left(T_{k}\right)=\varepsilon \bar{u}_{k}\left(r_{k}^{\left(l_{0}+1\right)}\right) / 2$ and hence can determine radii $s_{k}^{\left(l_{0}+1\right)}<\rho_{k}$ such that (64), (65) hold also for $l=l_{0}+1$, which on account of maximality of $l_{0}$ in view of Lemma 4.4 completes the concentration analysis.

If $\rho_{k}<\infty$, we may distinguish two cases. First assume that for some $L \geq 1$ there is a sequence $\left(x_{k}\right)$ such that $\rho_{k} / L \leq R_{k}\left(x_{k}\right) \leq\left|x_{k}\right| \leq L \rho_{k}$ and

$$
\lambda_{k}\left|x_{k}\right|^{4} u_{k}^{2}\left(x_{k}\right) e^{2 u_{k}^{2}\left(x_{k}\right)} \geq c_{0}>0 .
$$

By Proposition 4.1 we may assume that $\left|x_{k}\right|=\rho_{k}$.

Lemma 4.6 Assuming (69), we have $\bar{u}_{k}\left(\rho_{k}\right) / \bar{u}_{k}\left(r_{k}^{\left(l_{0}+1\right)}\right) \rightarrow 0$ as $k \rightarrow \infty$. Thus, for any $\varepsilon>0$ there is $T_{k}=T_{k}(\varepsilon) \in\left[r_{k}^{\left(l_{0}+1\right)}, \rho_{k}\right]$ such that $\bar{u}_{k}\left(T_{k}\right)=\varepsilon \bar{u}_{k}\left(r_{k}^{\left(l_{0}+1\right)}\right) / 2$.

Proof If we suppose that $\bar{u}_{k}\left(\rho_{k}\right) \geq \varepsilon \bar{u}_{k}\left(r_{k}^{\left(l_{0}+1\right)}\right) / 2$ for some $\varepsilon>0$, then, letting

$$
w_{k}(x)=w_{k}^{\left(l_{0}+1\right)}(x):=\bar{u}_{k}\left(r_{k}^{\left(l_{0}+1\right)}\right)\left(u_{k}(x)-\bar{u}_{k}\left(r_{k}^{\left(l_{0}+1\right)}\right)\right)
$$

and also writing $r_{k}=r_{k}^{\left(l_{0}+1\right)}$ for brevity, from (59) and the analogue of Lemma 2.4 for any constant $b<2$ we obtain

$$
\begin{aligned}
\lambda_{k}\left|x_{k}\right|^{4} u_{k}^{2}\left(x_{k}\right) e^{2 u_{k}^{2}\left(x_{k}\right)} & \leq C \lambda_{k} \rho_{k}^{4} \bar{u}_{k}^{2}\left(\rho_{k}\right) e^{2 \bar{u}_{k}^{2}\left(\rho_{k}\right)} \\
& \leq C \lambda_{k}\left(\frac{\rho_{k}}{r_{k}}\right)^{4} r_{k}^{4} \bar{u}_{k}^{2}\left(r_{k}\right) e^{2 \bar{u}_{k}^{2}\left(r_{k}\right)} e^{2\left(\bar{u}_{k}^{2}\left(\rho_{k}\right)-\bar{u}_{k}^{2}\left(r_{k}\right)\right)} \\
& \left.=C \tilde{e}_{k}\left(r_{k}\right)\right)\left(\frac{\rho_{k}}{r_{k}}\right)^{4} e^{2 \frac{\bar{u}_{k}\left(\rho_{k}\right)+\bar{u}_{k}\left(r_{k}\right)}{\bar{u}_{k}\left(r_{k}\right)} \bar{w}_{k}\left(\rho_{k}\right)} \\
& \leq C\left(\frac{\rho_{k}}{r_{k}}\right)^{4} e^{(2+\varepsilon) \bar{w}_{k}\left(\rho_{k}\right)} \leq C\left(\frac{\rho_{k}}{r_{k}}\right)^{4-(2+\varepsilon) b} \rightarrow 0
\end{aligned}
$$

as $k \rightarrow \infty$, if we choose $b<2$ such that $(2+\varepsilon) b>4$. This, however, contradicts our assumption (69).

Thus we can define numbers $s_{k}^{\left(l_{0}+1\right)}<\rho_{k}$ such that (64), (65) also hold for $l=l_{0}+1$. The concentration analysis at the scale $\rho_{k}=: r_{k}^{\left(l_{0}+2\right)}$ again uses a blow-up result.

Proposition 4.7 Assuming (69), there exist a finite set $S^{(0)} \subset \mathbb{R}^{4}$, a subsequence $\left(u_{k}\right)$, and a constant $c^{\left(l_{0}+2\right)}$ such that

$$
\eta_{k}^{\left(l_{0}+2\right)}(x):=\bar{u}_{k}\left(r_{k}^{\left(l_{0}+2\right)}\right)\left(u_{k}\left(r_{k}^{\left(l_{0}+2\right)} x\right)-\bar{u}_{k}\left(r_{k}^{\left(l_{0}+2\right)}\right)\right) \rightarrow \eta_{0}^{\left(l_{0}+2\right)}(x)
$$


locally $C^{3}$-uniformly on $\mathbb{R}^{4} \backslash S^{(0)}$ as $k \rightarrow \infty$, where $\eta^{\left(l_{0}+2\right)}=\eta_{0}^{\left(l_{0}+2\right)}+c^{\left(l_{0}+2\right)}$ extends to a solution of Eq. (7) of class $C^{\infty}\left(\mathbb{R}^{4}\right)$ with

$$
\int_{\mathbb{R}^{4}} 6 e^{4 \eta^{\left(l_{0}+2\right)}} \mathrm{d} x=\Lambda_{1} .
$$

From Proposition 4.7 the energy estimate at the scale $r_{k}^{\left(l_{0}+2\right)}$ follows as in the radial case and the concentration analysis at scales up to $\rho_{k}$ is complete. We then deal with secondary concentrations around $x_{k}^{(i)}=0$ at scales exceeding $\rho_{k}$.

Define

$$
X_{k, 1}=X_{k, 1}^{(i)}=\left\{x_{k}^{(j)} ; \exists C>0:\left|x_{k}^{(j)}\right| \leq C \rho_{k} \text { for all } k\right\}
$$

and denote as

$$
\rho_{k, 1}=\rho_{k, 1}^{(i)}=\frac{1}{2} \inf _{\left\{j ; x_{k}^{(j)} \notin X_{k, 1}\right\}}\left|x_{k}^{(j)}\right| .
$$

Again we set $\rho_{k, 1}=\infty$ if $\left\{j ; x_{k}^{(j)} \notin X_{k, 1}\right\}=\emptyset$. Note that $\rho_{k, 1} / \rho_{k} \rightarrow \infty$ as $k \rightarrow \infty$. Then (57)-(59) and the analogues of Lemmas 4.2-4.4, Lemma 4.6, and Lemma 2.4 hold for $r \in\left[L \rho_{k}, \rho_{k, 1}\right]$ for sufficiently large $L$, and we may continue as above to resolve concentrations in this range of scales.

In the remaining case when (69) fails to hold we have

$$
\lim _{L \rightarrow \infty} \limsup _{k \rightarrow \infty} \int_{\left\{x \in \Omega ; \rho_{k} / L \leq R_{k}(x) \leq|x| \leq L \rho_{k}\right\}} e_{k} \mathrm{~d} x=0 .
$$

Then either

$$
\lim _{L \rightarrow \infty} \limsup _{k \rightarrow \infty} N_{k}\left(L \rho_{k}, \rho_{k, 1} / L\right)=0,
$$

and we iterate to the next scale, or there exist radii $t_{k} \leq \rho_{k, 1}$ such that $t_{k} / \rho_{k} \rightarrow \infty$, $t_{k} / \rho_{k, 1} \rightarrow 0$ as $k \rightarrow \infty$ and a subsequence $\left(u_{k}\right)$ such that

$$
P_{k}\left(t_{k}\right) \geq c_{0}>0 \quad \text { for all } k \text {. }
$$

Lemma 4.8 Assume (71) holds true. Then $\bar{u}_{k}\left(t_{k}\right) / \bar{u}_{k}\left(r_{k}^{\left(l_{0}+1\right)}\right) \rightarrow 0$ as $k \rightarrow \infty$.

Proof Set $r_{k}=r_{k}^{\left(l_{0}+1\right)}$ for brevity and define $w_{k}=w_{k}^{\left(l_{0}+1\right)}$ as in the proof of Lemma 4.6. Going through the proof of Lemma 4.2, we note that (60) still holds for $r_{k} \leq r \leq t_{k}$. If we then suppose by contradiction that $\bar{u}_{k}\left(t_{k}\right) \geq \varepsilon \bar{u}_{k}\left(r_{k}\right) / 2$ for some $\varepsilon>0$ and sufficiently large $k$ a contradiction follows exactly as in the proof of Lemma 4.6.

By Lemma 4.8 and on account of (70), if we assume that (71) holds we can find numbers $s_{k}^{\left(l_{0}+1\right)}$ such that $s_{k}^{\left(l_{0}+1\right)} / \rho_{k} \rightarrow \infty$ as $k \rightarrow \infty$ and such that the analogues of (64), (65) hold for $l=l_{0}+1$ in the following sense. Similar to Lemma 4.2 we have

$$
\lim _{k \rightarrow \infty} \Lambda_{k}\left(s_{k}^{\left(l_{0}+1\right)}\right)=I_{1} \Lambda_{1}
$$

where $I_{1}$ is the total number of bubbles concentrating at the points $x_{k}^{(j)} \in X_{k, 1}^{(i)}$. Since we may repeat the previous argument with any $x_{k}^{(j)} \in X_{k, 1}^{(i)}$ in place of $x_{k}^{(i)}$ we also obtain the estimates 


$$
\begin{aligned}
& \lim _{L \rightarrow \infty} \lim _{k \rightarrow \infty}\left(\Lambda_{k}\left(s_{k}^{\left(l_{0}+1\right)}\right)-\sum_{x_{k}^{(j)} \in X_{k, 1}^{(i)}} \Lambda_{k}^{(j)}\left(L r_{k}^{\left(l_{0}^{(j)}+1\right)}\right)\right)=0, \\
& \lim _{k \rightarrow \infty} \frac{u_{k}\left(s_{k}^{\left(l_{0}+1\right)}\right)}{u_{k}\left(r_{k}^{\left(l_{0}^{(j)}+1\right)}\right)}=\lim _{k \rightarrow \infty} \frac{r_{k}^{\left(l_{0}^{(j)}+1\right)}}{s_{k}^{\left(l_{0}+1\right)}}=0 \quad \text { for all } x_{k}^{(j)} \in X_{k, 1}^{(i)},
\end{aligned}
$$

where $\Lambda_{k}^{(j)}(r)$ and $r_{k}^{\left(l_{0}^{(j)}+1\right)}$ are computed as above with reference to the concentration point $x_{k}^{(j)}$.

We then proceed by iteration. For $l \geq 2$ we inductively define the sets

$$
X_{k, l}=X_{k, l}^{(i)}=\left\{x_{k}^{(j)} ; \exists C>0:\left|x_{k}^{(j)}\right| \leq C \rho_{k, l-1} \text { for all } k\right\}
$$

and let

$$
\rho_{k, l}=\rho_{k, l}^{(i)}=\frac{1}{2} \inf _{\left\{j ; x_{k}^{(j)} \notin X_{k, l}^{(i)}\right\}}\left|x_{k}^{(j)}\right|,
$$

where $\rho_{k, l}=\infty$ if $\left\{j ; x_{k}^{(j)} \notin X_{k, l}^{(i)}\right\}=\emptyset$. Successively performing the above analysis at all scales $\rho_{k, l}$ and at all concentration points $x_{k}^{(j)}$, upon passing to further subsequences, if needed, we obtain Theorem 1.2.

\section{Proof of Proposition 4.1}

We argue indirectly, closely following the proof of Druet [4], Proposition 2. Let $y_{k} \in \Omega$ satisfy

$$
R_{k}\left(y_{k}\right) u_{k}\left(y_{k}\right)\left|\nabla u_{k}\left(y_{k}\right)\right|=\sup _{x \in \Omega} R_{k}(x) u_{k}(x)\left|\nabla u_{k}(x)\right|=: L_{k},
$$

and suppose by contradiction that $L_{k} \rightarrow \infty$ as $k \rightarrow \infty$. From (9) and elliptic regularity it follows that $s_{k}=R_{k}\left(y_{k}\right) \rightarrow 0$ as $k \rightarrow \infty$. Set

$$
\Omega_{k}=\left\{y ; y_{k}+s_{k} y \in \Omega\right\}
$$

and scale

$$
v_{k}(y)=u_{k}\left(y_{k}+s_{k} y\right), \quad y \in \Omega_{k} .
$$

Observe that (3), (4) again yield the uniform bounds

$$
\left\|\nabla v_{k}\right\|_{L^{4}} \leq C\left\|\nabla^{2} v_{k}\right\|_{L^{2}} \leq C, \int_{\Omega_{k}} v_{k} \Delta^{2} v_{k} \mathrm{~d} x \leq C .
$$

Letting $x_{k}^{(i)}$ be as in the statement of Theorem 1.1, we set

$$
y_{k}^{(i)}=\frac{x_{k}^{(i)}-y_{k}}{s_{k}}, \quad 1 \leq i \leq I,
$$


and let

$$
S_{k}=\left\{y_{k}^{(i)} ; 1 \leq i \leq I\right\} .
$$

Note that in the scaled coordinates we have

$$
\operatorname{dist}\left(0, S_{k}\right)=\inf \left\{\left|y_{k}^{(i)}\right| ; 1 \leq i \leq I\right\}=1
$$

and there holds

$$
\sup _{y \in \Omega}\left(\operatorname{dist}\left(y, S_{k}\right) v_{k}(y)\left|\nabla v_{k}(y)\right|\right) \leq v_{k}(0)\left|\nabla v_{k}(0)\right|=L_{k} \rightarrow \infty
$$

as $k \rightarrow \infty$; moreover, (9) implies the bound

$$
0 \leq v_{k} \Delta^{2} v_{k}=\lambda_{k} s_{k}^{4} v_{k}^{2} e^{2 v_{k}^{2}} \leq \frac{C}{\operatorname{dist}^{4}\left(y, S_{k}\right)} .
$$

Since $s_{k}=R_{k}\left(y_{k}\right) \rightarrow 0$ we may assume that as $k \rightarrow \infty$ the domains $\Omega_{k}$ exhaust a half-space

$$
\left.\Omega_{0}=\mathbb{R}^{3} \times\right]-\infty, R_{0}[
$$

where $0<R_{0} \leq \infty$. We also may assume that as $k \rightarrow \infty$ either $\left|y_{k}^{(i)}\right| \rightarrow \infty$ or $y_{k}^{(i)} \rightarrow y^{(i)}, 1 \leq i \leq I$, and we let $S_{0}$ be the set of accumulation points of $S_{k}$, satisfying $\operatorname{dist}\left(0, S_{0}\right)=1$. For $R>0$ denote as

$$
K_{R}=K_{k, R}=\Omega_{k} \cap B_{R}(0) \backslash \bigcup_{y \in S_{0}} B_{1 / R}(y) .
$$

Recalling that $\lambda_{k} s_{k}^{4} \rightarrow 0$, from (74), finally, for any $R>0$ we obtain

$$
\left\|\Delta^{2} v_{k}\right\|_{L^{\infty}\left(K_{R}\right)} \rightarrow 0 \quad \text { as } k \rightarrow \infty .
$$

We are now ready to show

Lemma 5.1 We have $R_{0}=\infty$; that is, $\Omega_{0}=\mathbb{R}^{4}$.

Proof Suppose by contradiction that $R_{0}<\infty$. Choosing $R=2 R_{0}$, from (73) and (2) we conclude the uniform bound

$$
\sup _{y \in K_{R}} \frac{v_{k}^{2}(y)}{v_{k}(0)\left|\nabla v_{k}(0)\right|} \leq C=C(R) .
$$

Letting $w_{k}=\frac{v_{k}}{\sqrt{v_{k}(0)\left|\nabla v_{k}(0)\right|}}$, we then have $0 \leq w_{k} \leq C$ while (72) and (75) give

$$
\left\|\nabla w_{k}\right\|_{L^{4}}+\left\|\nabla^{2} w_{k}\right\|_{L^{2}}+\left\|\Delta^{2} w_{k}\right\|_{L^{\infty}\left(K_{R}\right)} \rightarrow 0 \quad \text { as } k \rightarrow \infty .
$$

Since $w_{k}=0=\Delta w_{k}$ on $\partial \Omega_{k}$, it follows that $w_{k} \rightarrow 0$ in $C^{3, \alpha}\left(K_{R}\right)$, contradicting the fact that $w_{k}(0)\left|\nabla w_{k}(0)\right|=1$.

Lemma 5.2 As $k \rightarrow \infty$ we have $v_{k}(0) \rightarrow \infty$ and

$$
\frac{v_{k}}{v_{k}(0)} \rightarrow 1 \quad \text { in } C_{l o c}^{3, \alpha}\left(\mathbb{R}^{4} \backslash S_{0}\right) \text {. }
$$


Proof First observe that

$$
c_{k}:=\sup _{y \in B_{1 / 2}} v_{k}(y) \rightarrow \infty \quad \text { as } k \rightarrow \infty .
$$

Indeed, if by contradiction we assume that $c_{k} \leq C$, the uniform bounds (72) and (75) by elliptic regularity imply a uniform $C^{1}$-bound for $v_{k}$ near $y=0$, contradicting (73). Letting $w_{k}=c_{k}^{-1} v_{k}$, from (72) and (75) for any $R>0$ then we have

$$
\left\|\nabla w_{k}\right\|_{L^{4}}+\left\|\nabla^{2} w_{k}\right\|_{L^{2}}+\left\|\Delta^{2} w_{k}\right\|_{L^{\infty}\left(K_{R}\right)} \rightarrow 0 \quad \text { as } k \rightarrow \infty
$$

and we conclude that $w_{k}$ converges to a constant limit function $w$ in $C_{l o c}^{3, \alpha}\left(\mathbb{R}^{4} \backslash S_{0}\right)$. Recalling that $\operatorname{dist}\left(0, S_{0}\right)=1$, we obtain that

$$
w \equiv \sup _{y \in B_{1 / 2}} w(y)=\lim _{k \rightarrow \infty} \sup _{y \in B_{1 / 2}} w_{k}(y)=1 .
$$

In particular, we conclude that $c_{k}^{-1} v_{k}(0)=w_{k}(0) \rightarrow 1$ as $k \rightarrow \infty$ and therefore $v_{k}(0)=c_{k} w_{k}(0) \rightarrow \infty, \frac{v_{k}}{v_{k}(0)}=\frac{w_{k}}{w_{k}(0)} \rightarrow 1$ in $C_{l o c}^{3, \alpha}\left(\mathbb{R}^{4} \backslash S_{0}\right)$, as claimed.

Defining

$$
\tilde{v}_{k}(y)=\frac{v_{k}(y)-v_{k}(0)}{\left|\nabla v_{k}(0)\right|},
$$

from (73) and Lemma 5.2 with error $o(1) \rightarrow 0$ in $C_{l o c}^{3, \alpha}\left(\mathbb{R}^{4} \backslash S_{0}\right)$ as $k \rightarrow \infty$ we obtain the bound

$$
\left|\nabla \tilde{v}_{k}(y)\right|=\left(\frac{v_{k}(y)}{v_{k}(0)}+o(1)\right) \frac{\left|\nabla v_{k}(y)\right|}{\left|\nabla v_{k}(0)\right|} \leq \frac{1+o(1)}{\operatorname{dist}\left(y, S_{0}\right)} .
$$

Since $\tilde{v}_{k}(0)=0$, this shows that $\tilde{v}_{k}$ is uniformly bounded in $C^{1}\left(K_{R}\right)$ for any $R>0$. Moreover, from (74) and Lemma 5.2 with a constant $C=C(R)$ we obtain

$$
\Delta^{2} \tilde{v}_{k}=\frac{v_{k}(0)}{v_{k}} \frac{v_{k} \Delta^{2} v_{k}}{v_{k}(0)\left|\nabla v_{k}(0)\right|} \leq C \frac{v_{k}(0)}{L_{k} v_{k}} \rightarrow 0
$$

uniformly on $K_{R}$ as $k \rightarrow \infty$, for any $R>0$; the sequence $\left(\tilde{v}_{k}\right)$ thus is in fact bounded in $C_{l o c}^{3, \alpha}\left(\mathbb{R}^{4} \backslash S_{0}\right)$ and we may assume that $\tilde{v}_{k} \rightarrow \tilde{v}$ in $C_{l o c}^{3, \alpha}\left(\mathbb{R}^{4} \backslash S_{0}\right)$, where $\tilde{v}$ satisfies

$$
\Delta^{2} \tilde{v}=0, \quad \tilde{v}(0)=0, \quad|\nabla \tilde{v}(0)|=1, \quad|\nabla \tilde{v}(y)| \leq \frac{1}{\operatorname{dist}\left(y, S_{0}\right)} .
$$

Fix any point $x_{0} \in S_{0}$. For any $r>0$ by the divergence theorem we have

$$
\begin{aligned}
\int_{\partial B_{r}\left(x_{0}\right)} v_{k} \frac{x}{|x|} \cdot \nabla \Delta v_{k} \mathrm{~d} o & =\int_{B_{r}\left(x_{0}\right)}\left(v_{k} \Delta^{2} v_{k}+\nabla v_{k} \cdot \nabla \Delta v_{k}\right) \mathrm{d} x \\
& =\int_{B_{r}\left(x_{0}\right)}\left(v_{k} \Delta^{2} v_{k}-\left|\Delta v_{k}\right|^{2}\right) \mathrm{d} x+\int_{\partial B_{r}\left(x_{0}\right)} \frac{x}{|x|} \cdot \nabla v_{k} \Delta v_{k} \mathrm{~d} o .
\end{aligned}
$$

By (72) we can bound

$$
\left|\int_{B_{r}\left(x_{0}\right)}\left(v_{k} \Delta^{2} v_{k}-\left|\Delta v_{k}\right|^{2}\right) \mathrm{d} x\right| \leq C
$$


uniformly in $k$. The remaining term equals

$$
\int_{\partial B_{r}\left(x_{0}\right)} \frac{x}{|x|} \cdot \nabla v_{k} \Delta v_{k} \mathrm{~d} o=\left|\nabla v_{k}(0)\right| \int_{\partial B_{r}\left(x_{0}\right)} \frac{x}{|x|} \cdot \nabla \tilde{v}_{k} \Delta v_{k} \mathrm{~d} o .
$$

Since $\Delta v_{k} \leq 0$ by (2) and the maximum principle, the estimate (76) for sufficiently small $r>0$ yields the bound

$$
\left|\int_{\partial B_{r}\left(x_{0}\right)} \frac{x}{|x|} \cdot \nabla \tilde{v}_{k} \Delta v_{k} \mathrm{~d} o\right| \leq-(1+o(1)) r^{-1} \int_{\partial B_{r}\left(x_{0}\right)} \Delta v_{k} \mathrm{~d} o
$$

with error $o(1) \rightarrow 0$ as $k \rightarrow \infty$. By Fubini's theorem for any $r>0$ there is a subsequence $\left(u_{k}\right)$ and a radius $r / 2 \leq r_{0} \leq r$ such that there holds

$$
\left|r_{0} \int_{\partial B_{r_{0}}\left(x_{0}\right)} \Delta v_{k} \mathrm{~d} o\right| \leq 2 \int_{B_{r} \backslash B_{r / 2}\left(x_{0}\right)}\left|\Delta v_{k}\right| \mathrm{d} x \text { for all } k .
$$

From Hölder's inequality and (72) for such $r_{0}$ we conclude that

$$
\begin{aligned}
\left|r_{0}^{-1} \int_{\partial B_{r_{0}}\left(x_{0}\right)} \Delta v_{k} \mathrm{~d} o\right| & \leq 8 r^{-2} \int_{B_{r} \backslash B_{r / 2}\left(x_{0}\right)}\left|\Delta v_{k}\right| \mathrm{d} x \\
& \leq C\left(\int_{B_{r} \backslash B_{r / 2}\left(x_{0}\right)}\left|\Delta v_{k}\right|^{2} \mathrm{~d} x\right)^{1 / 2} \leq C .
\end{aligned}
$$

Replacing $r$ by $r_{0}$, from (81) for sufficiently large $k$ we then obtain

$$
\left|\int_{\partial B_{r_{0}}\left(x_{0}\right)} \frac{x}{|x|} \cdot \nabla v_{k} \Delta v_{k} \mathrm{~d} o\right| \leq C\left|\nabla v_{k}(0)\right| .
$$

Together with (79), (80) this estimate implies

$$
\left|\int_{\partial B_{r_{0}}\left(x_{0}\right)} v_{k} \frac{x}{|x|} \cdot \nabla \Delta v_{k} \mathrm{~d} o\right| \leq C\left(1+\left|\nabla v_{k}(0)\right|\right) .
$$

On the other hand, by Lemma 5.2 the integral on the left-hand side equals

$$
\int_{\partial B_{r_{0}}\left(x_{0}\right)} v_{k} \frac{x}{|x|} \cdot \nabla \Delta v_{k} \mathrm{~d} o=(1+o(1)) v_{k}(0)\left|\nabla v_{k}(0)\right| \int_{\partial B_{r_{0}}\left(x_{0}\right)} \frac{x}{|x|} \cdot \nabla \Delta \tilde{v}_{k} \mathrm{~d} o
$$

and we obtain that

$$
v_{k}(0)\left|\nabla v_{k}(0)\right|\left|\int_{\partial B_{r_{0}}\left(x_{0}\right)} \frac{x}{|x|} \cdot \nabla \Delta \tilde{v}_{k} \mathrm{~d} o\right| \leq C\left(1+\left|\nabla v_{k}(0)\right|\right) .
$$


But then by (73), and since by Lemma 5.2 we also have $v_{k}(0) \rightarrow \infty$, it follows that

$$
\int_{B_{r_{0}}\left(x_{0}\right)} \Delta^{2} \tilde{v}_{k} \mathrm{~d} x=\int_{\partial B_{r_{0}}\left(x_{0}\right)} \frac{x}{|x|} \cdot \nabla \Delta \tilde{v}_{k} \mathrm{~d} o \rightarrow 0
$$

as $k \rightarrow \infty$. Since $\Delta^{2} \tilde{v}_{k} \geq 0$, together with (77) this estimate implies that $\Delta^{2} \tilde{v}_{k} \rightarrow 0$ in $L_{l o c}^{1}\left(\mathbb{R}^{4}\right)$ as $k \rightarrow \infty$. The sequence $\left(\tilde{v}_{k}\right)$ therefore is uniformly locally bounded in $W^{2, q}$ for any $q<2$ and the limit $\tilde{v} \in W_{l o c}^{2, q}\left(\mathbb{R}^{4}\right)$ extends as a weakly biharmonic function to all of $\mathbb{R}^{4}$.

The function $\Delta \tilde{v} \in L_{l o c}^{q}\left(\mathbb{R}^{4}\right)$ then is weakly harmonic on $\mathbb{R}^{4}$. Since $\Delta \tilde{v} \leq 0$, from Harnack's inequality we conclude that $\Delta \tilde{v}$ everywhere equals a constant $c \leq 0$. If $c \neq 0$, as shown for instance in [1], Theorem 2.4, the function $\tilde{v}$ is a non-trivial quadratic polynomial, which violates the decay condition (78) for $\nabla \tilde{v}$. Thus we must have $c=0$ and $\tilde{v}$ is harmonic. The decay condition (78) then implies that $\tilde{v}$ is a constant; that is, $\tilde{v} \equiv \tilde{v}(0)=0$. But by (78) we have $|\nabla \tilde{v}(0)|=1$, which is the desired contradiction completing the proof of Proposition 4.1.

\section{Proof of Proposition 4.7}

As in Sect. 4, we fix an index $i \in\{1, \ldots, I\}$. Writing $r_{k}=r_{k}^{\left(l_{0}+2\right)}$ for simplicity, we define

$$
v_{k}(y)=u_{k}\left(x_{k}^{(i)}+r_{k} y\right), \quad y \in \Omega_{k},
$$

where by slight abuse of notation we denote as

$$
\Omega_{k}=\Omega_{k}^{(i)}=\left\{y ; x_{k}^{(i)}+r_{k} y \in \Omega\right\}
$$

the shifted and scaled set $\Omega$. For $j \in\{1, \ldots, I\}$ also let

$$
y_{k}^{(j)}=\frac{x_{k}^{(j)}-x_{k}^{(i)}}{r_{k}}
$$

and set

$$
S_{k}=S_{k}^{(i)}=\left\{y_{k}^{(j)} ; 1 \leq j \leq I\right\} .
$$

We may assume that as $k \rightarrow \infty$ either $\left|y_{k}^{(j)}\right| \rightarrow \infty$ or $y_{k}^{(j)} \rightarrow y^{(j)}, 1 \leq j \leq I$, and we let $S_{0}=S_{0}^{(i)}$ be the set of accumulation points of $S_{k}$. Note that $S_{0}$ contains the origin. Finally, let

$$
y_{k}^{(0)}=\frac{x_{k}-x_{k}^{(i)}}{r_{k}}
$$

be the scaled points $x_{k}$ for which (69) is valid, satisfying $\left|y_{k}^{(0)}\right|=1$. Again we may assume that $y_{k}^{(0)} \rightarrow y^{(0)}$ as $k \rightarrow \infty$. Observe that Proposition 4.1 implies the uniform bound

$$
\begin{aligned}
& \inf _{1 \leq j \leq I}\left|y-y_{k}^{(j)}\right| v_{k}(y)\left|\nabla v_{k}(y)\right| \\
& \quad=R_{k}\left(x_{k}^{(i)}+r_{k} y\right) u_{k}\left(x_{k}^{(i)}+r_{k} y\right)\left|\nabla u_{k}\left(x_{k}^{(i)}+r_{k} y\right)\right| \leq C .
\end{aligned}
$$


Since $v_{k}\left(y_{k}^{(0)}\right) \rightarrow \infty$ as $k \rightarrow \infty$ by (69), we conclude that $\left|\nabla v_{k}\right| \rightarrow 0$ as $k \rightarrow \infty$ locally uniformly on $\mathbb{R}^{4} \backslash S_{0}$ and therefore, since $\mathbb{R}^{4} \backslash S_{0}$ is connected, that

$$
v_{k}-v_{k}\left(y_{k}^{(0)}\right) \rightarrow 0 \quad \text { as } k \rightarrow \infty
$$

locally uniformly on $\mathbb{R}^{4} \backslash S_{0}$; moreover, as $k \rightarrow \infty$ the sets $\Omega_{k}$ exhaust all of $\mathbb{R}^{4}$.

For $\eta_{k}=\eta_{k}^{\left(l_{0}+2\right)}$ we then have the equation

$$
\begin{aligned}
\Delta^{2} \eta_{k}(y) & \left.=r_{k}^{4} v_{k}\left(y_{k}^{(0)}\right) \Delta^{2} u_{k},\left(x_{k}^{(i)}\right)+r_{k} y\right) \\
& =\lambda_{k} r_{k}^{4} v_{k}^{2}\left(y_{k}^{(0)}\right) e^{2 v_{k}^{2}\left(y_{k}^{(0)}\right)} \frac{v_{k}(y)}{v_{k}\left(y_{k}^{(0)}\right)} e^{2\left(v_{k}^{2}(y)-v_{k}^{2}\left(y_{k}^{(0)}\right)\right)} \\
& =\mu_{k} \hat{v}_{k}(y) e^{\left.2 \eta_{k}\left(\hat{v}_{k}(y)+1\right)\right)}
\end{aligned}
$$

where

$$
\mu_{k}=\lambda_{k} r_{k}^{4} v_{k}^{2}\left(y_{k}^{(0)}\right) e^{2 v_{k}^{2}\left(y_{k}^{(0)}\right)}=\lambda_{k}\left|x_{k}\right|^{4} u_{k}^{2}\left(x_{k}\right) e^{2 u_{k}^{2}\left(x_{k}\right)} \rightarrow \mu_{0}>0
$$

as $k \rightarrow \infty$ by (69), and where

$$
\hat{v}_{k}=\frac{v_{k}}{v_{k}\left(y_{k}^{(0)}\right)} \rightarrow 1 \quad \text { locally uniformly on } \mathbb{R}^{4} \backslash S_{0}
$$

on account of (84). The estimate (83) together with the condition $\bar{\eta}_{k}(1)=0$ also implies locally uniform $C^{1}$-bounds for $\left(\eta_{k}\right)$ away from $S_{0}$. By equation (85) then as $k \rightarrow \infty$ a subsequence $\left(\eta_{k}\right)$ converges locally $C^{3}$-uniformly on $\mathbb{R}^{4} \backslash S_{0}$ and weakly locally in $W^{2, q}$ to some limit $\eta_{0} \in W_{l o c}^{2, q}\left(\mathbb{R}^{4}\right)$ which is smooth away from $S_{0}$ and solves the equation $\Delta^{2} \eta_{0}=\mu_{0} e^{4 \eta_{0}}$ on $\mathbb{R}^{4} \backslash S_{0}$. Similar to (52), moreover, we can estimate

$$
\int_{\mathbb{R}^{4}} e^{4 \eta_{0}} \mathrm{~d} y \leq \lim _{L \rightarrow \infty} \liminf _{k \rightarrow \infty} \int_{B_{L} \backslash \bigcup_{y_{0} \in S_{0}} B_{1 / L}\left(y_{0}\right)} \hat{v}_{k}^{2} e^{\left.2 \eta_{k}\left(\hat{v}_{k}+1\right)\right)} \mathrm{d} y \leq C \Lambda,
$$

and $e^{4 \eta_{0}} \in L^{1}\left(\mathbb{R}^{4}\right)$. Taking account of (57), (65), and (67), we obtain the analogue of (48); that is,

$$
\int_{B_{1 / L}\left(y_{0}\right)} \Delta^{2} \eta_{k} \mathrm{~d} x \rightarrow 0
$$

for any $y_{0} \in S_{0}$, if we first let $k \rightarrow \infty$ and then pass to the limit $L \rightarrow \infty$. Thus, as in the proof of Proposition 2.3 we see that, in fact, $\eta_{0}$ solves the equation $\Delta^{2} \eta_{0}=\mu_{0} e^{4 \eta_{0}}$ on all of $\mathbb{R}^{4}$. For a suitably chosen constant $c_{0}$ the function $\eta=\eta_{0}+c_{0}$ then again solves Eq. (7) on $\mathbb{R}^{4}$. Finally, by (59) also (49) and hence the conclusion of Lemma 3.3 remain valid and we obtain the estimate (51). Again using Lin's [6] classification result we complete the proof of Proposition 4.7.

In the case of Proposition 4.5 we argue similarly, scaling with $r_{k}=r_{k}^{\left(l_{0}+1\right)}$. Note that $S_{0}=\{0\}$ in this case. 


\section{Concluding remarks}

Since positivity of $-\Delta u_{k}$ is only used near the blow-up points, it should not be too difficult to carry over our analysis to the case of Eq. (1) with Dirichlet boundary condition $u_{k}=\partial_{\nu} u_{k}=0$ on $\partial \Omega$ or to general nonlinearities of critical exponential growth, as studied in [2] or [4].

\section{References}

1. Adimurthi, Robert, F., Struwe, M.: Concentration phenomena for Liouville's equation in dimension four, J. Eur. Math. Soc. 8, 171-180 (2005)

2. Adimurthi, Struwe, M.: Global compactness properties of semilinear elliptic equations with critical exponential growth. J. Funct. Anal. 175, 125-167 (2000)

3. Adimurthi, Wei, J.: Blow up analysis of semilinear elliptic equations with critical growth in dimension 2. preprint (2005)

4. Druet, O.: Multibumps analysis in dimension 2. Duke Math J. 132(2), 217-269 (2006)

5. Hebey, E., Robert, F., Wen, Y.: Compactness and global estimates for a fourth order equation of critical Sobolev growth arising from conformal geometry. Comm. Contemp. Math. 8(1), 9-65 (2006)

6. Lin, C.S.: A classification of solutions of a conformally invariant fourth order equation in $\mathbb{R}^{n}$. Comm. Math. Helv. 73, 206-231 (1998)

7. Malchiodi, A.: Compactness of solutions to some geometric fourth-order equations. preprint (2005)

8. Malchiodi, A., Struwe, M.: The $Q$-curvature flow on $S^{4}$. J. Diff. Geom. preprint 73(1), 1-44 (2006)

9. Robert, F.: Quantization issues for fourth order equations with critical exponential growth. preprint (2005)

10. Robert, F., Struwe, M.: Asymptotic profile for a fourth order PDE with critical exponential growth in dimension four. Adv. Nonlinear Stud. 4(4), 397-415 (2004)

11. Wei, J.: Asymptotic behavior of a nonlinear fourth order eigenvalue problem. Comm. Partial Differential Equations 21(9-10), 1451-1467 (1996) 